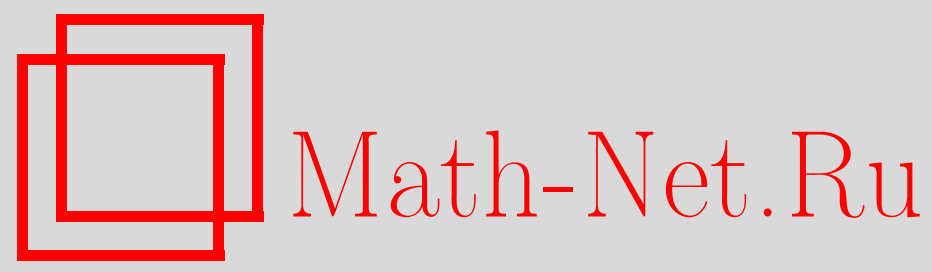

Г. Е. Иванов, Слабо выпуклые множества и их свойства, Матем. заметки, 2006, том 79, выпуск 1, 60-86

DOI: https://doi.org/10.4213/mzm2674

Использование Общероссийского математического портала Math-Net.Ru подразумевает, что вы прочитали и согласны с пользовательским соглашением http://www.mathnet.ru/rus/agreement

Параметры загрузки:

IP: 52.6 .47 .48

26 апреля 2023 г., 10:20:16

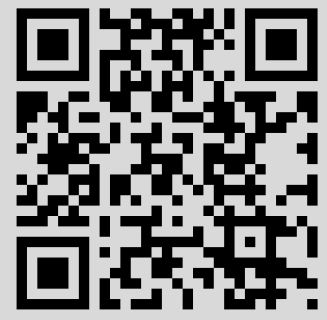




\title{
СЛАБО ВЫПУКЛЫЕ МНОЖЕСТВА И ИХ СВОЙСТВА
}

\author{
Г.Е. Иванов
}

\begin{abstract}
В работе введено понятие слабо выпуклых множеств. Приведены неулучшаемые оценки констант слабой выпук лости суммы и разности множеств. Доказано, что в гильбертовом пространстве гладкость множества эквивалентна слабой выпуклости этого множества и его дополнения. Здесь гладкость множества по определению означает, что на границе множества определено поле векторов единичных внешних нормалей, причем это векторное поле удовлетворяет условию Липшица. Получена теорема о минимаксе для одного класса задач с гладкими лебеговыми множествами целевой функции и сильно выпуклыми ограничениями. В качестве приложения полученных результатов доказана теорема об альтернативе для программных стратегий в линейной диффференциальной игре качества.

Библиограффия: 4 названия.
\end{abstract}

Развитая в последние годы теория сильно выпуклых множеств и функций позволила получить определенные условия регулярности в теории экстремальных задач, оптимальном управлении и теории дифференциальньх игр [1], [2]. В работах [3], [4] рассмотрены слабо выпуклые функции и, в частности, доказано, что гладкость (условие Липшица для градиента) функции $f(x)$ эквивалентна слабой вьпуклости функций $f(x)$ и $-f(x)$.

В настоящей работе введено понятие слабо выпуклых множеств. Приведены неулучшаемые оценки констант слабой вьпуклости суммы и разности множеств.

Доказано, что в гильбертовом пространстве гладкость множества эквивалентна слабой вьпуклости этого множества и его дополнения. Здесь гладкость множества по определению означает, что на границе множества определено полевекторов единичных внешних нормалей, причем это векторное поле удовлетворяет условию Липшица.

Получена теорема о минимаксе для одного класса задач с гладкими лебеговьми множествами целевой функции и сильно вьпукльми ограничениями. В качестве приложения полученных результатов доказана теорема об альтернативе для программных стратегий в линейной дифференциальной игре качества.

\section{1. Операции над множествами.}

ОПРЕДЕЛЕНИЕ. Пусть в линейном пространстве $\mathscr{L}$ заданы множества $X, Y \subset \mathscr{L}$ (здесь и далее, термин “множество” означает непустое множество). Геометрическими

Работа выполнена при финансовой поддержке Российского фонда фундаментальных исследований, грант № 03-01-14053. 
суммой и разностью или, что то же самое, суммой и разностью Минковского множеств $X$ и $Y$ назьваются соответственно множества

$$
X+Y=\{x+y: x \in X, y \in Y\}, \quad X * Y=\{z: z+Y \subset X\} .
$$

Произведением множества $X$ на число $\lambda$ называется множество

$$
\lambda X=\{\lambda x: x \in X\}
$$

Непосредственно из определения следует, что геометрическая разность $X *{ }^{*}-$ это пересечение сдвигов множества $X$ на векторы $d \in-Y$ :

$$
X *{ }^{*} Y=\bigcap_{d \in-Y}(X+d) .
$$

Условимся, что в выражениях, не содержащих скобок, геометрические операции сложения, вычитания и умножения на число выполняются слева направо; приоритет имеет операция умножения.

Заметим, что не все аналоги свойств операций сложения, вычитания и умножения чисел справедливы для множеств. Например, в общем случае равенство

$$
X \stackrel{*}{ } Z+Y=X+Y \stackrel{*}{ } Z
$$

не справедливо. Далее будут получены достаточные условия справедливости такого равенства. Кроме того, будет показано, что равенство $X * Z+Y=X+Y * Z$ означает существование седловой точки для некоторой минимаксной задачи.

Через $X^{c}$ будем обозначать дополнение множества $X$ :

$$
X^{c}=\{x \in \mathscr{L}: x \notin X\} .
$$

Непосредственно из определений вытекают следующие свойства геометрических операций:

1) $X+Y=Y+X$

2) $X+(Y+Z)=X+Y+Z$;

3) $X *{ }^{*}(Y+Z)=X \stackrel{*}{*} \stackrel{*}{Z}$;

4) a) $\lambda(X+Y)=\lambda X+\lambda Y$;

б) $\lambda(X \stackrel{*}{ } Y)=\lambda X \stackrel{*}{\lambda} Y$;

в) $(\lambda \mu) X=\lambda(\mu X)$;

5) $\left(X+d_{1}\right) *\left(Y+d_{2}\right)=X * * 2+d_{1}-d_{2}$ для любых векторов $d_{1}, d_{2} \in \mathscr{L}$;

6) a) $X \stackrel{*}{ } Y+Y \subset X$;

б) $X \subset X+Y * 2$

в) $Y \subset X *\left(X *{ }^{*} Y\right)$

7) а) если $X \subset Y$, то $\lambda X \subset \lambda Y$;

б) если $X_{1} \subset X_{2}, Y_{1} \subset Y_{2}$, то $X_{1}+Y_{1} \subset X_{2}+Y_{2}$;

в) если $X_{1} \subset X_{2}, Y_{1} \supset Y_{2}$, то $X_{1} * Y_{1} \subset X_{2} * Y_{2}$;

8) a) $X+(Y * 2) \subset X+Y * Z$;

б) $X+(Y * Z) \subset Y *(Z * X)$;

9) a) $X * * Y+Y * 4=X * Y$; 
б) $X+Y \stackrel{*}{ } Y+Y=X+Y$;

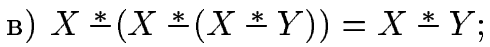

10) a) $\left(X \stackrel{*}{{ }^{c}}\right)^{c}=-Y+X^{c}$;

б) $(X+Y)^{c}=X^{c} \stackrel{*}{*}(-Y)$;

в) $X^{c} \stackrel{*}{Y^{c}}=-(Y \stackrel{*}{ } X)$;

11) $Y * *(Y \stackrel{*}{ } X)=X+(-Y)^{c} \stackrel{*}{ }(-Y)^{c}$;

12) a) $(X * Z) \cap(Y * Z)=(X \cap Y) * Z$;

б) $(X * * Y) \cap\left(X *{ }^{*}\right)=X *(Y \cup Z)$.

Перечисленные свойства геометрических операций будут свободно использоваться в дальнейшем изложении.

\section{2. Сильно и слабо выпуклые множества.}

ОПРЕДЕЛЕНИЕ. Пусть в топологическом векторном пространстве $\mathscr{L}$ задано множество $X$. Через $\operatorname{int} X, \operatorname{cl} X, \partial X$ будем обозначать соответственно внутренность, замькание и границу множества $X$.

ОПРЕДЕЛЕнИЕ. Через $\langle x, y\rangle$ будем обозначать скалярное произведение элементов $x$ и $y$ гильбертова пространства $\mathscr{H}$.

ОПРедЕЛЕниЕ. Через $\mathscr{B}_{R}$ будем обозначать шар радиуса $R \geqslant 0$ в банаховом пространстве $\mathscr{L}$ :

$$
\mathscr{B}_{R}=\{x \in \mathscr{L}:\|x\| \leqslant R\} .
$$

ОПРеДЕЛЕнИЕ. Множество $X \subset \mathscr{L}$ называется сильно выпуклым с константой $R>0$, если $\mathscr{B}_{R} * X \neq \varnothing$ и $\mathscr{B}_{R} *\left(\mathscr{B}_{R} * X\right)=X$.

Множество $X \subset \mathscr{L}$ назьвается слабо выпуклым с константой $R>0$, если $X \neq \mathscr{L}$ и $X+\operatorname{int} \mathscr{B}_{R}$ * int $\mathscr{B}_{R}=X$.

Множество $X \subset \mathscr{L}$ назьвается сильно (слабо) вогнутым с константой $R>0$, если $X \neq \mathscr{L}$ и множество $X^{c}$ сильно (слабо) выпукло с константой $R$.

Перечислим некоторые свойства слабо и сильно вьпукльх множеств.

ПрЕДЛОЖеНИЕ 1. Пусть в банаховом пространстве $\mathscr{L}$ задано множсество X. Пусть $R>0$. Следующие условия әквивалентны:

А) множество $X$ сильно выпукло с константой $R$;

В) существует множество $X_{1} \subset \mathscr{L}$ такое, ито $X=\mathscr{B}_{R} * X_{1}$;

C) для любой точки $x \in X^{c}$ найдется вектор $d \in \mathscr{L}$ такой, что $X \subset d+\mathscr{B}_{R}$ $u x \notin d+\mathscr{B}_{R}$.

ПРЕДЛОЖЕНИЕ 2. Пусть в банаховом пространстве $\mathscr{L}$ задано множество X. Пусть $R>0$. Следуюшие условия әквивалентны:

А) множество $X$ слабо выпукло с константой $R$;

B) $X \neq \mathscr{L}$ и существует множество $X_{1} \subset \mathscr{L}$ такое, что $X=X_{1} *$ int $\mathscr{B}_{R}$;

C) $X \neq \mathscr{L}$ и для любой точки $x \in X^{c}$ найдется вектор $d \in \mathscr{L}$ такой, что $x \in d+\operatorname{int} \mathscr{B}_{R} \subset X^{c}$. 
ПРЕДЛОЖЕНИЕ 3. Пусть в банаховом пространстве $\mathscr{L}$ задано множсество $X$. Пусть $R>0$. Следующие условия әквивалентны:

А) множество $X$ слабо вогнуто с константой $R$;

B) $X \neq \mathscr{L}$ и существует мнохество $X_{1} \subset \mathscr{L}$ такое, что $X=X_{1}+\operatorname{int} \mathscr{B}_{R}$;

C) $X \neq \mathscr{L}$ и для любой точки $x \in X$ найдется вектор $d \in \mathscr{L}$ такой, что $x \in d+\operatorname{int} \mathscr{B}_{R} \subset X$.

ПРЕДЛОЖЕНИЕ 4. Пусть в банаховом пространстве $\mathscr{L}$ задано множество $X$. Тогда

А) если множсество $X$ сильно или слабо выпукло с константой $R>0$, то мнохество $X$ замкнуто;

В) если множсество $X$ сильно или слабо вогнуто с константой $R>0$, то мнохсество $X$ открыто.

ПРЕДЛОЖЕНИЕ 5. Пусть в банаховом пространстве $\mathscr{L}$ множсество $X$ сильно (слабо) выпукло или сильно (слабо) вогнуто с константой $R>0$. Тогда

А) для любого вектора $d \in \mathscr{L}$ множество $d+X$ удовлетворяет тому жее условию;

В) для любого числа $\lambda \neq 0$ множество $\lambda X$ соответственно сильно (слабо) выпукло или сильно (слабо) вогнуто с константой $|\lambda| R$.

ПРЕДЛОЖЕНИЕ 6. Пусть заданы числа $r, R, 0<r<R, u$ множество $X \boldsymbol{в}$ банаховом пространстве. Тогда

А) если множество $X$ сильно выпукло (вогнуто) с константой $r$, то множество $X$ сильно выпукло (вогнуто) с константой $R$;

В) если мнохсество $X$ слабо выпукло (вогнуто) с константой $R$, то множество $X$ слабо выпукло (вогнуто) с константой $r$.

ПРЕДЛОЖЕНИЕ 7. Пусть в банаховом пространстве $\mathscr{L}$ заданы множества $X$, Y. Тогда

А) если множество $X$ сильно (слабо) выпукло с константой $R>0$ и $X$ * $Y$ $\neq \varnothing$, то множсество $X \stackrel{*}{Y}$ сильно (слабо) выпукло с константой $R$;

В) если множество $X$ сильно (слабо) вогнуто с константой $R>0 u X+Y$ $\neq \mathscr{L}$, то множество $X+Y$ сильно (слабо) вогнуто с константой $R$;

С) если множества $X, Y$ сильно (слабо) выпуклы с константой $R>0 u$ $X \cap Y \neq \varnothing$, то мнохсество $X \cap Y$ сильно (слабо) выпукло с константой $R$;

D) если мнохсества $X, Y$ сильно (слабо) вогнуты с константой $R>0 u$ $X \cup Y \neq \mathscr{L}$, то множсество $X \cup Y$ сильно (слабо) вогнуто с константой $R$.

ПРЕДЛОЖЕНИЕ 8. Пусть в гильбертовом пространстве задано множество $Х$. Пусть $R>0$. Следующие условия әквивалентны:

А) множсество $X$ сильно выпукло с константой $R$;

В) множество $X$ выпукло, замкнуто и существует выпуклое замкнутое множество $X_{1}$ mакое, что $X+X_{1}=\mathscr{B}_{R}$.

ПРЕДЛОЖЕНИЕ 9. Если в гильбертовом пространстве множества $X, Y$ сильно выпуклы с константами $R>0, r>0$ соответственно, то мнохсество $X+Y$ сильно выпукло с константой $R+r$. 
ПРЕДЛОЖЕНИЕ 10. Пусть в зильбертовом пространстве $\mathscr{H}$ множество $X$ сильно выпукло с константой $r>0$. Пусть заданы векторы $p \in \mathscr{H}, x \in X$ такие, что $\|p\|=1 u\langle p, x-a\rangle \geqslant 0$ для любого вектора $a \in X$. Тогда $X \subset x-r p+\mathscr{B}_{r}$.

Предложения 1-7 следуют непосредственно из определений и свойств геометрических операций с множествами. Доказательство предложений 8-10 можно найти, например, в [1].

Лемма 1. Пусть заданы числа $\delta, R_{1}, R_{2}$ и векторы $x_{0}, x_{1}, x_{2}$ гильбертова пространства $\mathscr{H}$. Пусть $\left\|x_{1}-x_{0}\right\| \leqslant R_{1}+\delta,\left\|x_{2}-x_{0}\right\| \leqslant R_{2}+\delta,\left\|x_{2}-x_{1}\right\| \geqslant R_{1}+R_{2}$. Тогда

$$
\left\|\left(R_{1}+R_{2}\right) x_{0}-R_{1} x_{2}-R_{2} x_{1}\right\|^{2} \leqslant\left(R_{1}+R_{2}\right)\left(4 R_{1} R_{2}+\left(R_{1}+R_{2}\right) \delta\right) \delta .
$$

ДОКАЗАТЕЛЬСТВо. Из условий леммы следует, что

$$
\begin{aligned}
\left(R_{1}+R_{2}\right)^{2} & \leqslant\left\|x_{2}-x_{1}\right\|^{2}=\left\|\left(x_{0}-x_{1}\right)-\left(x_{0}-x_{2}\right)\right\|^{2} \\
& =\left\|x_{0}-x_{1}\right\|^{2}+\left\|x_{0}-x_{2}\right\|^{2}-2\left\langle x_{0}-x_{1}, x_{0}-x_{2}\right\rangle .
\end{aligned}
$$

Поэтому

$$
\begin{aligned}
2\left\langle x_{0}-x_{1}, x_{0}-x_{2}\right\rangle & \leqslant\left\|x_{0}-x_{1}\right\|^{2}+\left\|x_{0}-x_{2}\right\|^{2}-\left(R_{1}+R_{2}\right)^{2} \\
& \leqslant\left(R_{1}+\delta\right)^{2}+\left(R_{2}+\delta\right)^{2}-\left(R_{1}+R_{2}\right)^{2} \\
& =-2 R_{1} R_{2}+2\left(R_{1}+R_{2}\right) \delta+2 \delta^{2} .
\end{aligned}
$$

Следовательно,

$$
\begin{aligned}
& \left\|\left(R_{1}+R_{2}\right) x_{0}-R_{1} x_{2}-R_{2} x_{1}\right\|^{2}=\left\|R_{1}\left(x_{0}-x_{2}\right)+R_{2}\left(x_{0}-x_{1}\right)\right\|^{2} \\
& \quad=R_{1}^{2}\left\|x_{0}-x_{2}\right\|^{2}+R_{2}^{2}\left\|x_{0}-x_{1}\right\|^{2}+2 R_{1} R_{2}\left\langle x_{0}-x_{1}, x_{0}-x_{2}\right\rangle \\
& \quad \leqslant R_{1}^{2}\left(R_{2}+\delta\right)^{2}+R_{2}^{2}\left(R_{1}+\delta\right)^{2}+2 R_{1} R_{2}\left(-R_{1} R_{2}+\left(R_{1}+R_{2}\right) \delta+\delta^{2}\right) \\
& \quad=\left(R_{1}+R_{2}\right)\left(4 R_{1} R_{2}+\left(R_{1}+R_{2}\right) \delta\right) \delta .
\end{aligned}
$$

Лемма 2. Пусть в гильбертовом пространстве $\mathscr{H}$ задано множество $A$, сильно выпуклое с константой $R_{1}>0$. Пусть заданы вектор $с \in \mathscr{H}$ и число $R_{2}>0$ такие, что $\left(c+\right.$ int $\left.\mathscr{B}_{R_{2}}\right) \cap A=\varnothing$. Тогда сущ,ствует вектор $b \in \mathscr{H}$ такой, что $A \subset b+\mathscr{B}_{R_{1}} u\|b-c\| \geqslant R_{1}+R_{2}$.

ДокАЗАТЕЛЬСтво. Из сильной вьпуклости множества $A$ следует выпуклость и замкнутость множества $A$. Поэтому существует вектор $x \in A$ такой, что $\|x-c\| \leqslant\|a-c\|$ для любого вектора $a \in A$. Из условия $\left(c+\operatorname{int} \mathscr{B}_{R_{2}}\right) \cap A=\varnothing$ следует, что $\|x-c\| \geqslant R_{2}$.

Определим вектор $p=(c-x) /\|c-x\|$. Покажем, что

$$
\langle p, x-a\rangle \geqslant 0 \quad \text { для любого вектора } a \in A \text {. }
$$

Пусть $a \in A$. Так как множество $A$ выпукло и $a \in A, x \in A$, то $x+t(a-x) \in A$ для любого $t \in(0 ; 1)$. Следовательно, для любого $t \in(0 ; 1)$ справедливо неравенство $\|x-c\| \leqslant\|x+t(a-x)-c\|$, т.е.

$$
\|x-c\|^{2} \leqslant\|x-c\|^{2}+2 t\langle x-c, a-x\rangle+t^{2}\|a-x\|^{2} .
$$

Поэтому $\langle x-c, a-x\rangle \geqslant 0$, т.е. $\langle p, x-a\rangle \geqslant 0$. Тем самым, доказано условие (1).

Из условия (1) в силу предложения 10 следует, что $A \subset x-R_{1} p+\mathscr{B}_{R_{1}}$. Определим вектор $b=x-R_{1} p$. Тогда

$$
\|b-c\|=\left\|x-c-R_{1} p\right\|=\left\|x-c-R_{1} \frac{c-x}{\|c-x\|}\right\|=\|x-c\|+R_{1} \geqslant R_{1}+R_{2} .
$$


Лемма 3. Пусть в гильбертовом пространстве $\mathscr{H}$ заданы множества $X, Y$. Пусть множество int $X$ слабо вогнуто с константой $R>0$, множество $X$ слабо выпукло с константой $\varrho>0, \operatorname{lint} X=X$. Пусть множество $Y$ сильно выпукло с константой $r \in(0 ; R)$. Пусть $z \in X \stackrel{*}{ } Y$,

$$
\sup \left\{\beta \geqslant 0: z+Y+\mathscr{B}_{\beta} \subset X\right\}=\gamma<R-r .
$$

Тогда $\partial X \cap\left(z+Y+\mathscr{B}_{\gamma}\right) \neq \varnothing$ и существуют числовая последовательность $\left\{\delta_{k}\right\}$ и последовательности $\left\{b_{k}\right\},\left\{d_{k}\right\}$ векторов пространства $\mathscr{H}$ такие, что $\delta_{k} \rightarrow 0$ при $k \rightarrow \infty$ и для любого $k \in \mathbb{N}$ справедливы условия

$$
z+Y \subset b_{k}+\mathscr{B}_{r}, \quad d_{k}+\operatorname{int} \mathscr{B}_{R} \subset \operatorname{int} X, \quad\left\|b_{k}-d_{k}\right\|<R-r-\gamma+\delta_{k} .
$$

ДокАЗАТЕЛЬСтво. Из слабой выпуклости множества $X$ следует замкнутость $X$. Отсюда и из определения супремума следует включение

$$
z+Y+\mathscr{B}_{\gamma} \subset X
$$

Из определения супремума также следует, что для любого $k \in \mathbb{N}$ вьполнено условие $z+Y+\mathscr{B}_{\gamma+1 / k} \not \subset X$. Поэтому существует последовательность векторов $\left\{a_{k}\right\}$ такая, что для любого $k \in \mathbb{N}$

$$
a_{k} \in\left(z+Y+\mathscr{B}_{\gamma+1 / k}\right) \cap X^{c} .
$$

Поскольку множество $X$ слабо вьпукло с константой $\varrho$, то для любого $k \in \mathbb{N}$ сушествует вектор $c_{k} \in \mathscr{H}$ такой, что

$$
a_{k} \in c_{k}+\operatorname{int} \mathscr{B}_{\varrho} \subset X^{c} .
$$

Из включений $(2),(4)$ следует, что $\left(c_{k}+\operatorname{int} \mathscr{B}_{\varrho}\right) \cap\left(z+Y+\mathscr{B}_{\gamma}\right)=\varnothing$. В силу пункта A $)$ предложения 5 и предложения 9 множество $z+Y+\mathscr{B}_{\gamma}$ сильно вьпукло с константой $r+\gamma$.

Зафиксируем произвольный номер $k \in \mathbb{N}$ и применим лемму 2 для множества $A=$ $z+Y+\mathscr{B}_{\gamma}$, вектора $c=c_{k}$ и чисел $R_{1}=r+\gamma, R_{2}=\varrho$. Получим, что для любого $k \in \mathbb{N}$ сушествует вектор $b_{k} \in \mathscr{H}$ такой, что

$$
z+Y+\mathscr{B}_{\gamma} \subset b_{k}+\mathscr{B}_{r+\gamma}
$$

и $\left\|b_{k}-c_{k}\right\| \geqslant \varrho+r+\gamma$.

Из включений (3), (5) следует, что $a_{k} \in b_{k}+\mathscr{B}_{r+\gamma+1 / k}$. Поэтому

$$
\left\|a_{k}-b_{k}\right\| \leqslant r+\gamma+\frac{1}{k}
$$

Из включения (4) следует, что $\left\|a_{k}-c_{k}\right\|<\varrho$. Применим лемму 1 для $x_{0}=a_{k}, x_{1}=b_{k}$, $x_{2}=c_{k}, R_{1}=r+\gamma, R_{2}=\varrho, \delta=1 / k$. Получим, что

$$
\left\|(\varrho+r+\gamma) a_{k}-(r+\gamma) c_{k}-\varrho b_{k}\right\|^{2} \leqslant(\varrho+r+\gamma)\left(4 \varrho(r+\gamma)+(\varrho+r+\gamma) \frac{1}{k}\right) \frac{1}{k} .
$$


Следовательно,

$$
\left\|(\varrho+r+\gamma) a_{k}-(r+\gamma) c_{k}-\varrho b_{k}\right\| \rightarrow 0 \quad \text { при } k \rightarrow \infty .
$$

Из (3) следует, что для любого $k \in \mathbb{N}$ существует вектор $y_{k} \in Y$ такой, что $\| z-a_{k}+$ $y_{k} \| \leqslant \gamma+1 / k$. Из $(2),(3)$ следует, что $a_{k} \notin z+Y+\mathscr{B}_{\gamma}$. Поэтому

$$
\gamma<\left\|z-a_{k}+y_{k}\right\| \leqslant \gamma+\frac{1}{k}
$$

Для любого $k \in \mathbb{N}$ определим вектор

$$
\widetilde{x}_{k}=z+y_{k}-\frac{\gamma}{\left\|z-a_{k}+y_{k}\right\|}\left(z-a_{k}+y_{k}\right) .
$$

Тогда $\widetilde{x}_{k} \in z+Y+\mathscr{B}_{\gamma} \subset X=\operatorname{clint} X$. Поэтому для любого $k \in \mathbb{N}$ существует вектор $x_{k} \in \operatorname{int} X$ такой, что $\left\|x_{k}-\widetilde{x}_{k}\right\|<1 / k$. Заметим, что

$$
\widetilde{x}_{k}-a_{k}=\left(1-\frac{\gamma}{\left\|z-a_{k}+y_{k}\right\|}\right)\left(z-a_{k}+y_{k}\right) \text {. }
$$

Следовательно, $\left\|\widetilde{x}_{k}-a_{k}\right\|=\left\|z-a_{k}+y_{k}\right\|-\gamma$. Отсюда и из соотношения (7) следует, что $\left\|\widetilde{x}_{k}-a_{k}\right\| \leqslant 1 / k$. Поэтому

$$
\left\|x_{k}-a_{k}\right\| \leqslant\left\|x_{k}-\widetilde{x}_{k}\right\|+\left\|\widetilde{x}_{k}-a_{k}\right\|<\frac{1}{k}+\frac{1}{k} .
$$

Таким образом,

$$
\left\|x_{k}-a_{k}\right\| \rightarrow 0 \quad \text { при } k \rightarrow \infty .
$$

Из включения $x_{k} \in \operatorname{int} X$ в силу слабой вогнутости множества int $X$ с константой $R$ получаем, что для любого $k \in \mathbb{N}$ существует вектор $d_{k} \in \mathscr{H}$ такой, что

$$
x_{k} \in d_{k}+\operatorname{int} \mathscr{B}_{R} \subset \operatorname{int} X .
$$

Из включений (4), (9) следует, что шары $c_{k}+\operatorname{int} \mathscr{B}_{\varrho}$ и $d_{k}+\operatorname{int} \mathscr{B}_{R}$ не пересекаются. Поэтому $\left\|d_{k}-c_{k}\right\| \geqslant R+\varrho$. Из включения (4) следует, что $\left\|a_{k}-c_{k}\right\|<\varrho$, а значит, $\left\|x_{k}-c_{k}\right\|<\varrho+\left\|x_{k}-a_{k}\right\|$. Из включения (9) следует, что $\left\|x_{k}-d_{k}\right\|<R$. Применим лемму 1 для $x_{0}=x_{k}, x_{1}=d_{k}, x_{2}=c_{k}, \delta=\left\|x_{k}-a_{k}\right\|, R_{1}=R, R_{2}=\varrho$. Получим неравенство

$$
\left\|(R+\varrho) x_{k}-R c_{k}-\varrho d_{k}\right\|^{2} \leqslant(R+\varrho)\left(4 R \varrho+(R+\varrho)\left\|x_{k}-a_{k}\right\|\right)\left\|x_{k}-a_{k}\right\| .
$$

Отсюда и из соотношения (8) следует, что

$$
\left\|(R+\varrho) x_{k}-R c_{k}-\varrho d_{k}\right\| \rightarrow 0 \quad \text { при } k \rightarrow \infty .
$$

Поскольку

$\varrho\left(b_{k}-d_{k}\right)=\left((R+\varrho) x_{k}-R c_{k}-\varrho d_{k}\right)-\left((\varrho+r+\gamma) x_{k}-\varrho b_{k}-(r+\gamma) c_{k}\right)-(R-r-\gamma)\left(x_{k}-c_{k}\right)$, 
то

$$
\varrho\left\|b_{k}-d_{k}\right\| \leqslant(R-r-\gamma)\left\|x_{k}-c_{k}\right\|+\Delta_{k},
$$

где $\Delta_{k}=\left\|(\varrho+r+\gamma) x_{k}-\varrho b_{k}-(r+\gamma) c_{k}\right\|+\left\|(R+\varrho) x_{k}-R c_{k}-\varrho d_{k}\right\|$. Из соотношений $(6),(8)$ следует, что $\left\|(\varrho+r+\gamma) x_{k}-\varrho b_{k}-(r+\gamma) c_{k}\right\| \rightarrow 0$ при $k \rightarrow \infty$. Отсюда и из (10) получаем, что

$$
\Delta_{k} \rightarrow 0 \quad \text { при } k \rightarrow \infty .
$$

Так как в силу включения (4) справедливы неравенства

$$
\left\|x_{k}-c_{k}\right\| \leqslant\left\|x_{k}-a_{k}\right\|+\left\|a_{k}-c_{k}\right\|<\varrho+\left\|x_{k}-a_{k}\right\|
$$

то $\varrho\left\|b_{k}-d_{k}\right\|<(R-r-\gamma)\left(\varrho+\left\|x_{k}-a_{k}\right\|\right)+\Delta_{k}$. Поэтому

$$
\left\|b_{k}-d_{k}\right\|<R-r-\gamma+\delta_{k}
$$

где

$$
\delta_{k}=\frac{1}{\varrho}\left((R-r-\gamma)\left\|x_{k}-a_{k}\right\|+\Delta_{k}\right) .
$$

Из (8), (11) следует, что $\delta_{k} \rightarrow 0$ при $k \rightarrow \infty$.

Из условий (3), (9) следует, что $a_{m} \notin d_{k}+\operatorname{int} \mathscr{B}_{R}$, а значит, $\left\|a_{m}-d_{k}\right\| \geqslant R$ для любых $k, m \in \mathbb{N}$. Из включений $(3),(5)$ следует, что $a_{m} \subset b_{k}+\mathscr{B}_{r+\gamma+1 / m}$. Поэтому $\left\|a_{m}-b_{k}\right\| \leqslant r+\gamma+1 / m$ для любых $k, m \in \mathbb{N}$. Применим лемму 1 для $x_{0}=b_{k}, x_{1}=d_{k}$, $x_{2}=a_{m}, R_{1}=R-r-\gamma, R_{2}=r+\gamma, \delta=\max \left\{\delta_{k}, 1 / m\right\}$. Получим соотношение $\left\|R b_{k}-(R-r-\gamma) a_{m}-(r+\gamma) d_{k}\right\| \rightarrow 0$ при $m \rightarrow \infty, k \rightarrow \infty$. Отсюда следует, что

$$
\begin{aligned}
& (R-r-\gamma)\left\|a_{m}-a_{k}\right\| \leqslant\left\|R b_{k}-(R-r-\gamma) a_{m}-(r+\gamma) d_{k}\right\| \\
& \quad+\left\|R b_{k}-(R-r-\gamma) a_{k}-(r+\gamma) d_{k}\right\| \rightarrow 0 \quad \text { при } m \rightarrow \infty, \quad k \rightarrow \infty
\end{aligned}
$$

Поэтому последовательность $\left\{a_{m}\right\}$ фундаментальна. В силу полноты пространства $\mathscr{H}$ последовательность $\left\{a_{m}\right\}$ сходится к некоторому вектору $a \in \mathscr{H}$.

Так как множество $z+Y+\mathscr{B}_{\gamma}$ сильно вьпукло с константой $r+\gamma$, оно замкнуто. Отсюда и из включения (3) следует, что $a \in z+Y+\mathscr{B} \gamma$. Так как $\left\|x_{k}-a\right\| \leqslant\left\|x_{k}-a_{k}\right\|+$ $\left\|a_{k}-a\right\| \rightarrow 0$ при $k \rightarrow \infty, x_{k} \in X, a_{k} \notin X$, то $a \in \partial X$. Поэтому $\partial X \cap\left(z+Y+\mathscr{B}_{\gamma}\right) \neq \varnothing$. Отсюда и из $(5),(9),(12)$ получаем утверждение леммы.

ТЕОРема 1 (о слабой вогнутости разности множеств). Пусть в гильбертовом пространстве $\mathscr{H}$ заданы мнохества $X, Y$. Пусть множество int $X$ слабо вогнуто с константой $R>0$, множество $X$ слабо выпукло с константой $\varrho>0$, clint $X=X$. Пусть мнохество $Y$ сильно выпукло с константой $r \in(0 ; R)$. Тог$\partial a$

A) мнохсество (int $X) * Y$ слабо вогнуто с константой $R-r$;

B) $(\operatorname{int} X) * Y=\operatorname{int}(X * Y)$;

C) $\operatorname{clint}\left(X *{ }^{*}\right)=X \stackrel{*}{ } Y$. 
ДокАЗАтЕльство. А) Зафиксируем произвольньй вектор $z \in(\operatorname{int} X) * Y$. Докажем существование вектора $d \in \mathscr{H}$ такого, что

$$
\|z-d\|<R-r \quad \text { и } \quad d+Y+\operatorname{int} \mathscr{B}_{R-r} \subset \operatorname{int} X .
$$

Определим число

$$
\gamma=\sup \left\{\beta \geqslant 0: z+Y+\mathscr{B}_{\beta} \subset X\right\} .
$$

Если $\gamma \geqslant R-r$, то $z+Y+\operatorname{int} \mathscr{B}_{R-r} \subset X$. В этом случае для вектора $d=z$ выполнены условия (13). Поэтому будем предполагать, что $\gamma<R-r$.

В силу леммы 3 справедливо равенство $\partial X \cap\left(z+Y+\mathscr{B}_{\gamma}\right) \neq \varnothing$ и сушествуют числовая последовательность $\left\{\delta_{k}\right\}$ и последовательности $\left\{b_{k}\right\},\left\{d_{k}\right\}$ векторов пространства $\mathscr{H}$ такие, что $\delta_{k} \rightarrow 0$ при $k \rightarrow \infty$ и для любого $k \in \mathbb{N}$ справедливы условия

$$
z+Y \subset b_{k}+\mathscr{B}_{r}, \quad d_{k}+\operatorname{int} \mathscr{B}_{R} \subset \operatorname{int} X, \quad\left\|b_{k}-d_{k}\right\|<R-r-\gamma+\delta_{k} .
$$

Так как $z \in(\operatorname{int} X) * Y$, то $z+Y \subset \operatorname{int} X$, а значит, $\partial X \cap(z+Y)=\varnothing$. Отсюда и из условия $\partial X \cap\left(z+Y+\mathscr{B}_{\gamma}\right) \neq \varnothing$ следует, что $\gamma>0$.

Отсюда и из неравенства $\left\|b_{k}-d_{k}\right\| \leqslant R-r-\gamma+\delta_{k}$ следует существование номера $n$ такого, что $\left\|b_{n}-d_{n}\right\|<R-r$. Определим вектор $d=d_{n}-b_{n}+z$. Тогда $\|z-d\|=\left\|b_{n}-d_{n}\right\|<R-r$. Из включений $z+Y \subset b_{n}+\mathscr{B}_{r}, d_{n}+$ int $\mathscr{B}_{R} \subset \operatorname{int} X$ следует, что

$$
d+Y+\operatorname{int} \mathscr{B}_{R-r} \subset d+b_{n}-z+\mathscr{B}_{r}+\operatorname{int} \mathscr{B}_{R-r}=d_{n}+\operatorname{int} \mathscr{B}_{R} \subset \operatorname{int} X .
$$

Поэтому условие (13) вьполнено.

Тем самым, для любого вектора $z \in(\operatorname{int} X) * Y$ доказано существование вектора $d \in \mathscr{H}$ такого, что $z \in d+\operatorname{int} \mathscr{B}_{R-r} \subset(\operatorname{int} X) * Y$. Отсюда и из предложения 3 следует пункт А) теоремы.

В) Из пункта В) предложения 4 в силу слабой вогнутости множества (int $X) * Y$ следует, что это множество открыто. Отсюда следует, что

$$
(\operatorname{int} X) \stackrel{*}{Y}=\operatorname{int}((\operatorname{int} X) \stackrel{*}{ } Y) \subset \operatorname{int}(X \stackrel{*}{Y}) \text {. }
$$

Докажем обратное включение: $\operatorname{int}(X * Y) \subset(\operatorname{int} X) * Y$. Пусть $z \in \operatorname{int}(X * Y)$; тогда существует число $\varepsilon>0$ такое, что $z+\operatorname{int} \mathscr{B}_{\varepsilon} \subset X * Y$. Следовательно, $z+Y+$ $\operatorname{int} \mathscr{B}_{\varepsilon} \subset X$. Поэтому $z+Y \subset \operatorname{int} X$, а значит, $z \in(\operatorname{int} X) * Y$.

С) Из замкнутости множества $X$ следует замкнутость множества $X \stackrel{*}{Y}$. Поэтому

$$
\operatorname{clint}(X \stackrel{*}{*}) \subset \operatorname{cl}(X \stackrel{*}{*})=X \stackrel{*}{ } Y .
$$

Покажем, что обратное включение $X * Y \subset \operatorname{clint}(X * Y)$ также выполнено. Пусть $z \in X *{ }^{*} Y$. Требуется доказать, что $z \in \operatorname{clint}\left(X *{ }^{*}\right)$. Определим число

$$
\gamma=\sup \left\{\beta \geqslant 0: z+Y+\mathscr{B}_{\beta} \subset X\right\} \text {. }
$$

Если $\gamma>0$, то $z+Y \subset \operatorname{int} X$. Следовательно, $z \in(\operatorname{int} X) * Y=\operatorname{int}\left(X *{ }^{*} Y\right) \subset$ $\operatorname{clint}(X * Y)$. Поэтому в случае $\gamma>0$ включение $z \in \operatorname{clint}\left(X * *^{\prime}\right)$ выполнено. Остается рассмотреть случай $\gamma=0$. 
В силу леммы 3 существуют числовая последовательность $\left\{\delta_{k}\right\}$ и последовательности $\left\{b_{k}\right\},\left\{d_{k}\right\}$ векторов пространства $\mathscr{H}$ такие, что $\delta_{k} \rightarrow 0$ при $k \rightarrow \infty$ и для любого $k \in \mathbb{N}$ справедливы условия

$$
z+Y \subset b_{k}+\mathscr{B}_{r}, \quad d_{k}+\operatorname{int} \mathscr{B}_{R} \subset \operatorname{int} X, \quad\left\|b_{k}-d_{k}\right\|<R-r+\delta_{k} .
$$

Для любого $k \in \mathbb{N}$ определим вектор

$$
f_{k}= \begin{cases}\frac{\delta_{k}}{\left\|d_{k}-b_{k}\right\|}\left(d_{k}-b_{k}\right), & \text { если } d_{k} \neq b_{k}, \\ 0, & \text { если } d_{k}=b_{k} .\end{cases}
$$

Тогда $\left\|f_{k}\right\| \leqslant \delta_{k} \rightarrow 0$ при $k \rightarrow \infty$. Заметим, что

$$
\left\|b_{k}-d_{k}+f_{k}\right\|=\left\|b_{k}-d_{k}\right\|-\delta_{k}<R-r .
$$

Поэтому $b_{k}+f_{k}-d_{k} \in \operatorname{int} \mathscr{B}_{R-r}$. Отсюда и из включений

$$
z+Y \subset b_{k}+\mathscr{B}_{r}, \quad d_{k}+\operatorname{int} \mathscr{B}_{R} \subset \operatorname{int} X
$$

следует, что

$$
z+f_{k}+Y \subset b_{k}+f_{k}+\mathscr{B}_{r} \subset d_{k}+\operatorname{int} \mathscr{B}_{R} \subset \operatorname{int} X .
$$

Следовательно, $z+f_{k} \in(\operatorname{int} X) * Y=\operatorname{int}(X * Y)$. Поэтому $z \in \operatorname{clint}(X * Y)$.

ЗАмечание 1 . Если $X=\mathscr{B}_{R}, Y=\mathscr{B}_{r}, 0<r<R$, то (int $\left.X\right) *{ }^{*} Y=\operatorname{int} \mathscr{B}_{R-r}$. Этот пример показьвает неулучшаемость оценки константы $R-r$ слабой вогнутости множества $(\operatorname{int} X) * Y$ при условиях, что множество int $X$ слабо вогнуто с константой $R$, а множество $Y$ сильно вьпукло с константой $r$.

ЗАмЕчаниЕ 2. Согласно теореме 1 константа $\varrho$ слабой вьпуклости множества $X$ не влияет на константу $R-r$ слабой вогнутости множества (int $X) * Y$. Тем не менее, для слабой вогнутости множества (int $X) * Y$ слабая вьпуклость множества $X$ существенна. Действительно, рассмотрим в $\mathbb{R}^{2}$ множества $Y=\mathscr{B}_{1}, X=\left(\bar{x}_{1}+\mathscr{B}_{2}\right) \cup\left(\bar{x}_{2}+\mathscr{B}_{2}\right)$, где

$$
\bar{x}_{1}=\left\|\begin{array}{c}
-1 \\
0
\end{array}\right\|, \quad \bar{x}_{2}=\left\|\begin{array}{l}
1 \\
0
\end{array}\right\|,
$$

$\mathscr{B}_{1}, \mathscr{B}_{2}$ - замкнутые шары радиусов 1 и 2 с центром в точке $\overline{0}$. Поскольку множество int $X$ является объединением двух открытых шаров радиуса 2 , согласно пункту D) предложения 7 множество int $X$ слабо вогнуто с константой $R=2$. Множество $Y$ силь-

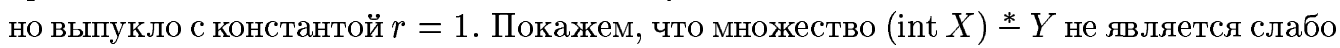
выпукльм с константой $R-r=1$. Определим вектор

$$
\bar{x}_{3}=\left\|\begin{array}{c}
0 \\
1 / 2
\end{array}\right\| \text {. }
$$

Так как множество int $X$ содержит шар с центром в точке $\bar{x}_{3}$ и радиусом $\sqrt{3}-1 / 2>1$, то $\bar{x}_{3} \in(\operatorname{int} X) *{ }^{*} Y$. Покажем, что не существует вектора $d \in \mathbb{R}^{2}$ такого, что $\bar{x}_{3} \in d+$ int $\mathscr{B}_{1} \subset(\operatorname{int} X) * Y$. Предположим, что такой вектор $d$ существует. Тогда $\left\|\bar{x}_{3}-d\right\|<1$ и $d+$ int $\mathscr{B}_{2} \subset X$. Из включения $d+\operatorname{int} \mathscr{B}_{2} \subset X$ следует, что $d=\bar{x}_{1}$ или $d=\bar{x}_{2}$. Но тогда неравенство $\left\|\bar{x}_{3}-d\right\|<1$ невьполнено. Поэтому в силу предложения 3 множество (int $X) \stackrel{*}{Y}$ не является слабо выпуклым с константой $R-r=1$. 
ЗАмЕчАниЕ 3. Если множество $Y$ является компактом, то, как легко видеть, множество (int $X) *{ }^{*} Y$ открыто. Отсюда, повторяя доказательство пункта В) теоремы 1 , легко получить равенство $\operatorname{int}\left(X *{ }^{*} Y\right)=(\operatorname{int} X) \stackrel{*}{Y}$. Поскольку в конечномерном пространстве сильно вьпуклое множество является компактом, в конечномерном пространстве для сильно вьпуклого множества $Y$ и произвольного множества $X$ справедливо равенство $\operatorname{int}(X * Y)=(\operatorname{int} X) * Y$. В бесконечномерном пространстве это равенство в общем случае не справедливо. Действительно, рассмотрим, например, в пространстве $\ell_{2}$ множества

$$
X=\left\{x=\left(x_{1}, x_{2}, \ldots\right) \in \ell_{2}: \sum_{k=1}^{\infty}\left(1+\frac{1}{k}\right)^{-2} x_{k}^{2}<1\right\}, \quad Y=\mathscr{B}_{1}
$$

Тогда множество $X$ открыто, $X *{ }^{*} Y=\{\overline{0}\}, \operatorname{int}\left(X *{ }^{*} Y\right)=\varnothing,(\operatorname{int} X) *{ }^{*}=\{\overline{0}\}$. Поэтому равенство $\operatorname{int}(X * Y)=(\operatorname{int} X) * Y$ не вьполнено.

ТЕОРема 2 (о слабой вьпуклости суммы множеств). Пусть в гильбертовом пространстве $\mathscr{H}$ заданы мнохества $X, Y$. Пусть мнохество $X$ слабо выпукло $c$ константой $R>0$, множество int $X$ слабо вогнуто $c$ константой $\varrho>0$, clint $X=X$. Пусть мнохсество $Y$ сильно выпукло с константой $r \in(0 ; R)$. Тогда

А) мнохсество $X+Y$ слабо выпукло с константой $R-r$;

B) $\operatorname{int}(X+Y)=(\operatorname{int} X)+Y$;

C) $\operatorname{clint}(X+Y)=X+Y$.

ДокАЗАтЕльство. Определим множества $X_{1}=(\operatorname{int} X)^{c}, Y_{1}=-Y$. Так как множество $X$ слабо выпукло с константой $R$, множество int $X_{1}=\operatorname{int}(\operatorname{int} X)^{c}=(\operatorname{cl} \operatorname{int} X)^{c}$ $=X^{c}$ слабо вогнуто с константой $R$. Из слабой вогнутости множества int $X$ с константой $\varrho$ следует слабая выпуклость множества $X_{1}$. Так как множество $Y$ сильно вьпукло с константой $r$, множество $Y_{1}$ обладает тем же свойством. В силу теоремы 1 множество $\left(\operatorname{int} X_{1}\right) * Y_{1}$ слабо вогнуто с константой $R-r$ и справедливы равенства

$$
\left(\operatorname{int} X_{1}\right) * Y_{1}=\operatorname{int}\left(X_{1} * Y_{1}\right), \quad \operatorname{clint}\left(X_{1} * Y_{1}\right)=X_{1} * Y_{1} .
$$

Из слабой вогнутости множества (int $\left.X_{1}\right) * Y_{1}=X^{c} * Y_{1}$ с константой $R-r$ следует слабая выпуклость множества $\left(X^{c} * Y_{1}\right)^{c}=X+Y$ с константой $R-r$.

Из равенства $\left(\operatorname{int} X_{1}\right) * Y_{1}=\operatorname{int}\left(X_{1} * Y_{1}\right)$ следует, что

$$
\left(\left(\operatorname{int} X_{1}\right) * Y_{1}\right)^{c}=\left(\operatorname{int}\left(X_{1} * Y_{1}\right)\right)^{c},
$$

т.e. $X+Y=\operatorname{cl}((\operatorname{int} X)+Y)$.

Из равенства $\operatorname{clint}\left(X_{1} * Y_{1}\right)=X_{1} * Y_{1}$ следует равенство

$$
\left(\operatorname{clint}\left(X_{1} * Y_{1}\right)\right)^{c}=\left(X_{1} * Y_{1}\right)^{c}
$$

т.e. int $\operatorname{cl}((\operatorname{int} X)+Y)=(\operatorname{int} X)+Y$.

Отсюда и из равенства $X+Y=\operatorname{cl}((\operatorname{int} X)+Y)$ следует, что int $(X+Y)=(\operatorname{int} X)+Y$, а значит, $X+Y=\operatorname{cl}((\operatorname{int} X)+Y)=\operatorname{clint}(X+Y)$. 


\section{3. Слабая выпуклость и гладкость множеств.}

Лемма 4. Пусть в гильбертовом пространстве $\mathscr{H}$ задано множество $X$ такое, что мнохество $\mathrm{cl} X$ слабо выпукло с константой $R_{1}>0$, а множсство int $X$ слабо вогнуто с константой $R_{2}>0$. Пусть int $\operatorname{cl} X=\operatorname{int} X, \operatorname{cl}$ int $X=\operatorname{cl} X$. Тогда для любой граничной точки $x \in \partial X$ существует вектор $p=p(x) \in \mathscr{H}$ такой, что

$$
\|p\|=1, \quad x+R_{1} p+\operatorname{int} \mathscr{B}_{R_{1}} \subset \operatorname{int}\left(X^{c}\right), \quad x-R_{2} p+\operatorname{int} \mathscr{B}_{R_{2}} \subset \operatorname{int} X .
$$

ДокАЗАтЕЛьСтво. Пусть $x \in \partial X$. Так как $x \in(\operatorname{int} X)^{c}=(\operatorname{int} \operatorname{cl} X)^{c}=\operatorname{cl}\left((\operatorname{cl} X)^{c}\right)$, для любого $k \in \mathbb{N}$ существует вектор $x_{k} \in(\operatorname{cl} X)^{c}$ такой, что $\left\|x_{k}-x\right\|<1 / k$. Поэтому в силу слабой выпуклости множества $\mathrm{cl} X$ с константой $R_{1}$ существует вектор $a_{k} \in \mathscr{H}$ такой, что $x_{k} \in a_{k}+\operatorname{int} \mathscr{B}_{R_{1}} \subset(\operatorname{cl} X)^{c}$. Следовательно, $\left\|a_{k}-x\right\|<R_{1}+1 / k$.

Аналогично в силу слабой вогнутости множества int $X$ с константой $R_{2}$ и включения $x \in \operatorname{cl} X=\operatorname{clint} X$ получаем, что для любого $n \in \mathbb{N}$ существует вектор $b_{n}$ такой, что $b_{n}+\operatorname{int} \mathscr{B}_{R_{2}} \subset \operatorname{int} X$ и $\left\|b_{n}-x\right\|<R_{2}+1 / n$.

Так как шары $a_{k}+\operatorname{int} \mathscr{B}_{R_{1}}$ и $b_{n}+\operatorname{int} \mathscr{B}_{R_{2}}$ не пересекаются, то $\left\|a_{k}-b_{n}\right\| \geqslant R_{1}+R_{2}$ для любых $k, n \in \mathbb{N}$. Применяя лемму 1 для $x_{0}=x, x_{1}=a_{k}, x_{2}=b_{n}, \delta=\max \{1 / k, 1 / n\}$, получим, что

$$
\left\|\left(R_{1}+R_{2}\right) x-R_{1} b_{n}-R_{2} a_{k}\right\| \rightarrow 0 \quad \text { при } k \rightarrow \infty, \quad n \rightarrow \infty
$$

Отсюда следует, что

$$
R_{2}\left\|a_{n}-a_{k}\right\| \leqslant\left\|\left(R_{1}+R_{2}\right) x-R_{1} b_{n}-R_{2} a_{n}\right\|+\left\|\left(R_{1}+R_{2}\right) x-R_{1} b_{n}-R_{2} a_{k}\right\| \rightarrow 0
$$

при $k \rightarrow \infty, n \rightarrow \infty$. Поэтому последовательность $\left\{a_{k}\right\}$ фундаментальна. Аналогично доказьвается фундаментальность последовательности $\left\{b_{n}\right\}$. В силу полноты пространства $\mathscr{H}$ последовательности $\left\{a_{k}\right\}$ и $\left\{b_{n}\right\}$ сходятся к некоторым векторам $a$ и $b$ соответственно. Обозначим $p=(x-b) / R_{2}$. Тогда $b=x-R_{2} p$. Из соотношения (14) следует, что $\left(R_{1}+R_{2}\right) x-R_{1} b-R_{2} a=0$. Поэтому $a=x+R_{1} p$.

Покажем, что $b+\operatorname{int} \mathscr{B}_{R_{2}} \subset \operatorname{int} X$. Предположим противное: сушествует вектор $z \in b+\operatorname{int} \mathscr{B}_{R_{2}}, z \notin \operatorname{int} X$. Из включения $b_{n}+\operatorname{int} \mathscr{B}_{R_{2}} \subset \operatorname{int} X$ следует, что $\left\|b_{n}-z\right\| \geqslant R_{2}$. Отсюда и из соотношения $b_{n} \rightarrow b$ при $n \rightarrow \infty$ следует, что $\|b-z\| \geqslant R_{2}$, что противоречит условию $z \in b+\operatorname{int} \mathscr{B}_{R_{2}}$. Аналогично, из соотношений $a_{k}+\operatorname{int} \mathscr{B}_{R_{1}} \subset(\operatorname{cl} X)^{c}$ и $a_{k} \rightarrow a$ при $k \rightarrow \infty$ следует, что $a+\operatorname{int} \mathscr{B}_{R_{1}} \subset \operatorname{int}\left(X^{c}\right)$.

ЛЕмма 5. Пусть в гильбертовом пространстве $\mathscr{H}$ задано мнохсество Х. Пусть для любой точки $x \in \partial X$ существует вектор $p=p(x) \in \mathscr{H}$ такой, что $\|p\|=1, x+R p+\operatorname{int} \mathscr{B}_{R} \subset \operatorname{int}\left(X^{c}\right), x-R p+\operatorname{int} \mathscr{B}_{R} \subset \operatorname{int} X$. Tогда функиия $p: \partial X \rightarrow \mathscr{H}$ удовлетворяет условию Липшица с константой $1 / R$, т.е. для любых точек $x_{1}, x_{2} \in \partial X$ справедливо неравенство

$$
\left\|p\left(x_{1}\right)-p\left(x_{2}\right)\right\| \leqslant \frac{1}{R}\left\|x_{1}-x_{2}\right\| .
$$


ДоказАТЕЛЬСтво. Пусть $x_{1}, x_{2} \in \partial X$. Обозначим $d=x_{1}-x_{2}, p_{1}=p\left(x_{1}\right)$, $p_{2}=p\left(x_{2}\right)$. Так как $\left(x_{1}+R p_{1}+\operatorname{int} \mathscr{B}_{R}\right) \cap\left(x_{2}-R p_{2}+\operatorname{int} \mathscr{B}_{R}\right)=\varnothing$, то

$$
\left\|R p_{1}+R p_{2}+d\right\| \geqslant 2 R .
$$

Tак как $\left(x_{1}-R p_{1}+\operatorname{int} \mathscr{B}_{R}\right) \cap\left(x_{2}+R p_{2}+\operatorname{int} \mathscr{B}_{R}\right)=\varnothing$, то

$$
\left\|R p_{1}+R p_{2}-d\right\| \geqslant 2 R \text {. }
$$

Следовательно,

$$
R^{2}\left\|p_{1}+p_{2}\right\|^{2}+\|d\|^{2} \geqslant 4 R^{2}=2 R^{2}\left\|p_{1}\right\|^{2}+2 R^{2}\left\|p_{2}\right\|^{2} .
$$

Поэтому $\|d\|^{2} \geqslant R^{2}\left\|p_{1}-p_{2}\right\|^{2}$, а значит,

$$
\left\|p_{1}-p_{2}\right\| \leqslant \frac{1}{R}\left\|x_{1}-x_{2}\right\| \text {. }
$$

ОПРЕДЕЛЕНИЕ. Пусть в банаховом пространстве $\mathscr{L}$ заданы множество $X$ и точка $x_{0}$. Контингентным конусом (конусом Булигана) называется множество

$$
T_{X}\left(x_{0}\right)=\bigcap_{\varepsilon>0} \bigcap_{\delta>0} \bigcup_{t \in(0 ; \delta)}\left(\frac{1}{t}\left(X-x_{0}\right)+\mathscr{B}_{\varepsilon}\right) .
$$

Непосредственно из определения контингентного конуса следует

ПРЕДЛОЖЕНИЕ 11. Пусть в банаховом пространстве $\mathscr{L}$ заданы множество $X$ u точка $x_{0}$. Вектор $v \in \mathscr{L}$ принадлежит конусу $T_{X}\left(x_{0}\right)$ тогда и только тогда, когда существуют числовая последовательность $\left\{\lambda_{k}\right\}$ и последовательность $\left\{v_{k}\right\}$ әлементов пространства $\mathscr{L}$ такие, что $\lambda_{k} \rightarrow 0, v_{k} \rightarrow v$ nрu $k \rightarrow \infty u \lambda_{k}>0$, $x_{0}+\lambda_{k} v_{k} \in X$ для любого $k \in \mathbb{N}$.

ОПРЕДЕЛЕниЕ. Пусть в гильбертовом пространстве $\mathscr{H}$ заданы множество $X$ и точка $x_{0}$. Вектор $p_{0} \in \mathscr{H}$ назьвается единичным вектором внешней нормали множества $X$ в точке $x_{0}$, если

$$
\left\|p_{0}\right\|=1, \quad T_{X}\left(x_{0}\right)=\left\{x \in \mathscr{H}:\left\langle p_{0}, x\right\rangle \leqslant 0\right\} \quad \text { и } T_{X^{c}}\left(x_{0}\right)=\left\{x \in \mathscr{H}:\left\langle p_{0}, x\right\rangle \geqslant 0\right\} .
$$

Непосредственно из определения следует, что вектор $p_{0}$ является вектором внешней нормали множества $X$ в точке $x_{0}$ тогда и только тогда, когда вектор $-p_{0}$ является вектором внешней нормали множества $X^{c}$ в точке $x_{0}$.

ОПРЕДЕЛЕНИЕ. Пусть заданы число $L>0$ и множество $X$ в гильбертовом пространстве $\mathscr{H}$. Будем говорить, что множество $X$ является телесно-гладким с константой $L$, если $\partial X \neq \varnothing$ и существует функция $p: \partial X \rightarrow \mathscr{H}$ (мы ее будем назьвать полем единичных нормалей множества $X$ ) такая, что

А) для любой граничной точки $x \in \partial X$ вектор $p(x)$ является единичньм вектором внешней нормали множества $X$ в точке $x$ и

В) функция $p(x)$ удовлетворяет условию Липшица на множестве $\partial X$ с константой $L$ :

$$
\left\|p\left(x_{1}\right)-p\left(x_{2}\right)\right\| \leqslant L\left\|x_{1}-x_{2}\right\| \quad \text { для любых } x_{1}, x_{2} \in \partial X .
$$


Заметим, что граница телесно-гладкого множества является гладким многообразием коразмерности 1. Однако из того, что граница множества $X$ является гладким многообразием коразмерности 1 еще не следует, что множество $X$ является телесно-гладким. Например, множество $X=\{x \in \mathscr{H}:\|x\| \neq 1\}$ не является телесно-гладким, так как для любой точки $x \in \partial X$ контингентньй конус $T_{X}(x)$ совпадает со всем пространством $\mathscr{H}$, а значит, не существует единичного вектора внешней нормали. Однако граница множества $X$ - это единичная сфера $\partial X=\{x \in \mathscr{H}:\|x\|=1\}$, которая является гладким многообразием коразмерности 1.

ЛЕмма 6. Пусть числовое множество $X \subset \mathbb{R}$ является телесно-гладким $c$ константой $1 / R, R>0$. Пусть $p_{0}$ - единичный вектор внешней нормали множества $X$ в точке $x_{0} \in \partial X$. Тогда справедливо включение $x_{0}-R p_{0}+\operatorname{int} \mathscr{B}_{R} \subset X$.

ДокАЗАТЕЛЬСТВо. Определим $r_{0}$ как супремум чисел $r>0$ таких, что $\left(x_{0}-r p_{0}-r\right.$; $\left.x_{0}-r p_{0}+r\right) \subset X$. В случае $r_{0} \geqslant R$ имеем

$x_{0}-R p_{0}+\operatorname{int} \mathscr{B}_{R}=\left(x_{0}-R p_{0}-R ; x_{0}-R p_{0}+R\right) \subset\left(x_{0}-r_{0} p_{0}-r_{0} ; x_{0}-r_{0} p_{0}+r_{0}\right) \subset X$.

Поэтому в данном случае утверждение леммы справедливо.

Покажем, что случай $r_{0}<R$ не реализуется. Предположим, что $r_{0}<R$. Из определения вектора внешней нормали следует, что $r_{0}>0$. Определим число $x_{1}=x_{0}-2 r_{0} p_{0}$. Из определения супремума следует, что $x_{1} \in \partial X$. Пусть $p_{1}-$ единичный вектор внешней нормали множества $X$ в точке $x_{1}$. Из включения $\left(x_{1}+r_{0} p_{0}-r_{0} ; x_{1}+r_{0} p_{0}+r_{0}\right) \subset X$ и определения вектора внешней нормали получаем, что $p_{1}=-p_{0}$. Отсюда следует, что $\left|p_{1}-p_{0}\right|=2\left|p_{0}\right|=2$. Поскольку множество $X$ является телесно-гладким с константой $1 / R$, то

$$
\left|p_{1}-p_{0}\right| \leqslant \frac{1}{R}\left|x_{1}-x_{0}\right| .
$$

Следовательно, $\left|x_{1}-x_{0}\right| \geqslant 2 R$, т.е. $r_{0} \geqslant R$, что противоречит предположению $r_{0}<R$.

Лемма 7. Пусть в гильбертовом пространстве $\mathscr{H}$ множество $X$ является телесно-гладким с константой $1 / R, R>0$. Пусть $p_{0}-$ единичный вектор внешней нормали множсества $X$ в точке $x_{0} \in \partial X$. Тогда справедливо включение

$$
\left(x_{0}-R p_{0}+\operatorname{int} \mathscr{B}_{R}\right) \cap\left(x_{0}+\mathscr{B}_{R / 2}\right) \subset X .
$$

ДокАЗАТЕЛЬСтво. Предположим противное: существует вектор $x_{1} \in\left(x_{0}-R p_{0}+\right.$ int $\left.\mathscr{B}_{R}\right) \cap\left(x_{0}+\mathscr{B}_{R / 2}\right), x_{1} \notin X$.

Если $\mathscr{L}$ - одномерное пространство, то утверждение леммы следует из леммы 6 . Иначе существует вектор $\tau_{0} \in \mathscr{H}$ такой, что $\left\|\tau_{0}\right\|=1,\left\langle p_{0}, \tau_{0}\right\rangle=0$ и $x_{1}-x_{0}$ является линейной комбинацией векторов $\tau_{0}, p_{0}$. Будем предполагать, что $\left\langle\tau_{0}, x_{1}-x_{0}\right\rangle \geqslant 0$. Если это предположение не вьполняется, то заменим $\tau_{0}$ на $-\tau_{0}$ и оно станет вьполняться. Тогда существует число $\alpha \in[0 ; 2 \pi)$ такое, что

$$
x_{1}-x_{0}=\left\|x_{1}-x_{0}\right\|\left(\tau_{0} \sin \alpha-p_{0} \cos \alpha\right) .
$$

Поскольку $x_{1} \in x_{0}-R p_{0}+\operatorname{int} \mathscr{B}_{R}$, то $\left\|x_{0}-x_{1}-R p_{0}\right\|<R$. Следовательно,

$$
\left\|x_{0}-x_{1}\right\|^{2}<2 R\left\langle p_{0}, x_{0}-x_{1}\right\rangle
$$


т.е. $\left\|x_{0}-x_{1}\right\|^{2}<2 R\left\|x_{0}-x_{1}\right\| \cos \alpha$. Поэтому $0<\left\|x_{0}-x_{1}\right\|<2 R \cos \alpha$. Из неравенства $\left\langle\tau_{0}, x_{1}-x_{0}\right\rangle \geqslant 0$ следует, что $\sin \alpha \geqslant 0$. Отсюда и из неравенства $0<\left\|x_{0}-x_{1}\right\|<2 R \cos \alpha$ получаем $\alpha \in[0 ; \pi / 2)$.

Определим

$$
\psi=\arccos \frac{\left\|x_{0}-x_{1}\right\|}{2 R}-\alpha .
$$

Из неравенства $\left\|x_{0}-x_{1}\right\|<2 R \cos \alpha$ следует, что

$$
\arccos \frac{\left\|x_{0}-x_{1}\right\|}{2 R}>\alpha \text {. }
$$

Поэтому $\psi \in(0 ; \pi / 2)$.

Определим векторы

$$
p_{1}=p_{0} \cos \psi+\tau_{0} \sin \psi, \quad \tau_{1}=-p_{0} \sin \psi+\tau_{0} \cos \psi .
$$

Заметим, что $\left\|p_{1}\right\|=\left\|\tau_{1}\right\|=1,\left\langle p_{1}, \tau_{1}\right\rangle=0$.

Поскольку

$$
\begin{aligned}
\left\|x_{0}-x_{1}-R p_{1}\right\|^{2}-R^{2} & =\left\|x_{0}-x_{1}\right\|^{2}+2 R\left\langle p_{1}, x_{1}-x_{0}\right\rangle \\
& =\left\|x_{0}-x_{1}\right\|^{2}+2 R\left\|x_{0}-x_{1}\right\|(-\cos \alpha \cos \psi+\sin \alpha \sin \psi) \\
& =\left\|x_{0}-x_{1}\right\|^{2}-2 R\left\|x_{0}-x_{1}\right\| \cos (\alpha+\psi) \\
& =\left\|x_{0}-x_{1}\right\|^{2}-2 R\left\|x_{0}-x_{1}\right\| \frac{\left\|x_{0}-x_{1}\right\|}{2 R}=0,
\end{aligned}
$$

то $\left\|x_{0}-x_{1}-R p_{1}\right\|=R$. Отсюда и из того, что вектор $x_{0}-x_{1}-R p_{1}$ является линейной комбинацией векторов $\tau_{1}, p_{1}$ следует, что существует $\varphi_{1} \in[0 ; 2 \pi)$ :

$$
x_{0}-x_{1}-R p_{1}=-R p_{1} \cos \varphi_{1}-R \tau_{1} \sin \varphi_{1} .
$$

Так как

$R \sin \varphi_{1}=\left\langle x_{1}-x_{0}, \tau_{1}\right\rangle=\left\|x_{0}-x_{1}\right\|(\cos \alpha \sin \psi+\sin \alpha \cos \psi)=\left\|x_{0}-x_{1}\right\| \sin (\alpha+\psi)>0$, то $\varphi_{1} \in(0 ; \pi)$. Из условия $x_{1} \in x_{0}+\mathscr{B}_{R / 2}$ следует, что

$$
\begin{aligned}
\frac{1}{4} R^{2} & \geqslant\left\|x_{1}-x_{0}\right\|^{2}=\left\|R p_{1}\left(1-\cos \varphi_{1}\right)+R \tau_{1} \sin \varphi_{1}\right\|^{2} \\
& =R^{2}\left(1-\cos \varphi_{1}\right)^{2}+R^{2} \sin ^{2} \varphi_{1}=R^{2}\left(2-2 \cos \varphi_{1}\right) .
\end{aligned}
$$

Поэтому $\cos \varphi_{1} \geqslant 1-1 / 8>\sqrt{2} / 2$. Отсюда и из включения $\varphi_{1} \in(0 ; \pi)$ получаем $\varphi_{1} \in(0 ; \pi / 4)$.

Определим функцию

$$
x(\varphi)=x_{0}-R p_{1}(1-\cos \varphi)+R \tau_{1} \sin \varphi .
$$

Из равенства $x_{0}-x_{1}-R p_{1}=-R p_{1} \cos \varphi_{1}-R \tau_{1} \sin \varphi_{1}$ следует, что $x_{1}=x\left(\varphi_{1}\right)$. Через $\varphi_{2}$ обозначим инфимум таких $\varphi>0$, что $x(\varphi) \notin X$. Поскольку $x\left(\varphi_{1}\right)=x_{1} \notin X$, то $\varphi_{2} \leqslant \varphi_{1}$, а значит, $\varphi_{2} \in[0 ; \pi / 4)$. 
Рассмотрим случай $\varphi_{2}=0$. В этом случае по определению супремума существует последовательность положительных чисел $\left\{\lambda_{k}\right\}$, стремящаяся к нулю и такая, что $x\left(\lambda_{k}\right) \notin X$. Поскольку

$$
\frac{1}{R \lambda_{k}}\left(x\left(\lambda_{k}\right)-x_{0}\right) \rightarrow \tau_{1} \quad \text { при } k \rightarrow \infty,
$$

в силу предложения 11 получаем $\tau_{1} \in T_{X^{c}}\left(x_{0}\right)$. Отсюда и из определения вектора внешней нормали следует неравенство $\left\langle p_{0}, \tau_{1}\right\rangle \geqslant 0$. Поэтому $\sin \psi \leqslant 0$, что противоречит включению $\psi \in(0 ; \pi / 2)$. Полученное противоречие показывает, что $\varphi_{2} \neq 0$, т.е. $\varphi_{2} \in(0 ; \pi / 4)$.

Обозначим $x_{2}=x\left(\varphi_{2}\right)$. Для любого $k \in \mathbb{N}$ определим вектор

$$
v_{k}=\frac{2 k}{\varphi_{2}}\left(x_{2}-x\left(\varphi_{2}-\frac{\varphi_{2}}{2 k}\right)\right), \quad v=x^{\prime}\left(\varphi_{2}\right) \text {. }
$$

Так как для любого $\varphi \in\left(0 ; \varphi_{2}\right)$ справедливо включение $x(\varphi) \in X$, то $x\left(\varphi_{2}-\varphi_{2} /(2 k)\right) \in X$ для любого $k \in \mathbb{N}$. Из соотношений

$$
x_{2}-\frac{\varphi_{2}}{2 k} v_{k}=x\left(\varphi_{2}-\frac{\varphi_{2}}{2 k}\right) \in X, \quad v_{k} \rightarrow v \text { при } k \rightarrow \infty,
$$

в силу предложения 11 получаем $-v \in T_{X}\left(x_{2}\right)$. Так как $x_{2} \in \partial X$, по условию леммы сушествует $p_{2}$ - единичньй вектор внешней нормали множества $X$ в точке $x_{2}$. Из условия $-v \in T_{X}\left(x_{2}\right)$ следует неравенство $\left\langle p_{2}, v\right\rangle \geqslant 0$, где

$$
v=x^{\prime}\left(\varphi_{2}\right)=R \tau_{1} \cos \varphi_{2}-R p_{1} \sin \varphi_{2}=R \tau_{0} \cos \left(\varphi_{2}+\psi\right)-R p_{0} \sin \left(\varphi_{2}+\psi\right)
$$

Обозначим через $\overline{p_{2}}$ ортогональную проекцию вектора $p_{2}$ на линейную оболочку векторов $\tau_{0}, p_{0}$. Определим число $\beta \in[-\pi ; \pi)$ из условия $\overline{p_{2}}=\left(p_{0} \cos \beta+\tau_{0} \sin \beta\right)\left\|\overline{p_{2}}\right\|$. Тогда

$$
0 \leqslant\left\langle p_{2}, v\right\rangle=\left\langle\overline{p_{2}}, v\right\rangle=\left\|\overline{p_{2}}\right\| R \sin \left(\beta-\varphi_{2}-\psi\right) .
$$

Следовательно, $\sin \left(\beta-\varphi_{2}-\psi\right) \geqslant 0$.

Так как поле единичных нормалей удовлетворяет условию Липшица на множестве $\partial X$ с константой $1 / R$, то

$$
\left\|p_{2}-p_{0}\right\| \leqslant \frac{1}{R}\left\|x_{2}-x_{0}\right\|
$$

Следовательно, $R^{2}\left\|p_{2}-p_{0}\right\|^{2} \leqslant\left\|x_{2}-x_{0}\right\|^{2}$, т.е. $R^{2}\left(2-2\left\|\overline{p_{2}}\right\| \cos \beta\right) \leqslant R^{2}\left(2-2 \cos \varphi_{2}\right)$. Поэтому $\left\|\overline{p_{2}}\right\| \cos \beta \geqslant \cos \varphi_{2}$. Отсюда и из условий $\beta \in[-\pi ; \pi), \varphi_{2} \in[0 ; \pi / 4],\left\|\overline{p_{2}}\right\| \leqslant 1$ следует неравенство $|\beta| \leqslant \varphi_{2}$, а значит, $\beta-\varphi_{2} \in[-\pi / 2 ; 0]$. Поэтому в силу условия $\psi \in(0 ; \pi / 2)$ справедливо включение $\beta-\varphi_{2}-\psi \in(-\pi ; 0)$, что противоречит неравенству $\sin \left(\beta-\varphi_{2}-\psi\right) \geqslant 0$.

Лемма 8. Пусть заданы векторы $и, v$ гильбертова пространства $\mathscr{H}$ и числа $r>0, \delta>0$ такие, что $r \leqslant\|u\| \leqslant r+\delta,\|v\|<r$. Тогда существует число $\theta \in(0 ; 1)$ такое, что для вектора $w=\theta u+(1-\theta) v$ справедливы неравенства $\|w\|<r,\|u-w\|^{2}<2(r+\delta) \delta$. 
ДокАЗАТЕЛЬСтво. Из неравенств $\|v\|<r \leqslant\|u\|$ следует, что $u \neq v$. Определим число $t_{0}=\langle u, u-v\rangle /\|u-v\|^{2}$, вектор $y=u-t_{0}(u-v)$ и функцию $x(t)=y+t(u-v)$ при $t \in \mathbb{R}$.

Из соотношений $2\langle u, u-v\rangle=\|u\|^{2}-\|v\|^{2}+\|u-v\|^{2}>0$ следует неравенство $t_{0}>0$. Так как $\langle y, u-v\rangle=\langle u, u-v\rangle-t_{0}\|u-v\|^{2}=0$, то для любого $t \in \mathbb{R}$ справедливо равенство

$$
\|x(t)\|^{2}=\|y\|^{2}+t^{2}\|u-v\|^{2} .
$$

Поскольку $x\left(t_{0}-1\right)=y+t_{0}(u-v)-u+v=v$, то $\left\|x\left(t_{0}-1\right)\right\|^{2}=\|v\|^{2}<r^{2}$. Поэтому согласно (15)

$$
\|y\|^{2} \leqslant\left\|x\left(t_{0}-1\right)\right\|^{2}<r^{2} .
$$

Определим число $t_{1}=\sqrt{r^{2}-\|y\|^{2}} /\|u-v\|$. Из неравенства $\|y\|^{2}<r^{2}$ следует, что $t_{1}>0$.

Заметим, что

$$
\left\|x\left(t_{1}\right)\right\|^{2}=\|y\|^{2}+t_{1}^{2}\|u-v\|^{2}=r^{2} .
$$

Отсюда и из неравенств $\|v\|<r,\|u\| \leqslant r+\delta$ следуют неравенства

$$
\left\|x\left(t_{1}\right)\right\|^{2}>\|v\|^{2} \quad \text { и } \quad\left\|x\left(t_{1}\right)\right\|^{2} \geqslant r^{2}+\|u\|^{2}-(r+\delta)^{2}>\|u\|^{2}-2(r+\delta) \delta .
$$

Следовательно, в силу непрерьвности функции $x\left(t_{1}\right)$ существует число $t_{2} \in\left(0 ; t_{1}\right)$ такое, что

$$
\left\|x\left(t_{2}\right)\right\|^{2}>\|v\|^{2} \quad \text { и } \quad\left\|x\left(t_{2}\right)\right\|^{2}>\|u\|^{2}-2(r+\delta) \delta .
$$

Обозначим $w=x\left(t_{2}\right)$. Из равенства (15) следует, что

$$
\|w\|^{2}=\|y\|^{2}+t_{2}^{2}\|u-v\|^{2}<\|y\|^{2}+t_{1}^{2}\|u-v\|^{2}=\left\|x\left(t_{1}\right)\right\|^{2}=r^{2} .
$$

Поэтому

$$
\|w\|<r .
$$

Из соотношений $\left\|x\left(t_{0}\right)\right\|=\|u\| \geqslant r,\left\|x\left(t_{1}\right)\right\|=r$ и (15) следует, что

$$
\|y\|^{2}+t_{0}^{2}\|u-v\|^{2} \geqslant r^{2}=\|y\|^{2}+t_{1}^{2}\|u-v\|^{2} .
$$

Поэтому $t_{0}^{2} \geqslant t_{1}^{2}$. Отсюда и из неравенств $t_{0}>0, t_{2}<t_{1}$ получаем неравенства $t_{2}<t_{1}<t_{0}$.

Из неравенства $\left\|x\left(t_{2}\right)\right\|^{2}>\|v\|^{2}$ и равенств $x\left(t_{0}-1\right)=v,(15)$ получаем, что $\left(t_{0}-1\right)^{2}$ $<t_{2}^{2}$. Отсюда и из неравенства $t_{2}>0$ следует неравенство $t_{0}-1<t_{2}<t_{0}$. Поэтому существует число $\theta \in(0 ; 1)$ такое, что $t_{2}=(1-\theta)\left(t_{0}-1\right)+\theta t_{0}$, а значит,

$$
w=x\left(t_{2}\right)=(1-\theta) x\left(t_{0}-1\right)+\theta x\left(t_{0}\right)=(1-\theta) v+\theta u .
$$

Так как $w=y+t_{2}(u-v),\langle y, u-v\rangle=0$, то $\langle w, u-v\rangle=t_{2}\|u-v\|^{2}$. Отсюда и из соотношений

$$
u-w=u-x\left(t_{2}\right)=u-y-t_{2}(u-v)=\left(t_{0}-t_{2}\right)(u-v)
$$

следует, что

$$
\langle w, u-w\rangle=\left(t_{0}-t_{2}\right)\langle w, u-v\rangle=\left(t_{0}-t_{2}\right) t_{2}\|u-v\|^{2}>0
$$

Поэтому

$$
\|u\|^{2}=\|w+u-w\|^{2}=\|w\|^{2}+\|u-w\|^{2}+2\langle w, u-w\rangle>\|w\|^{2}+\|u-w\|^{2} .
$$

Отсюда и из неравенства (16) получаем

$$
\|u-w\|^{2}<\|u\|^{2}-\|w\|^{2}=\|u\|^{2}-\left\|x\left(t_{2}\right)\right\|^{2}<2(r+\delta) \delta,
$$

что вместе с (17), (18) завершает доказательство. 
ЛЕмма 9. Пусть в гильбертовом пространстве $\mathscr{H}$ множество $X$ является телесно-гладким с константой $1 / R, R>0$. Пусть заданы векторы $a, x \in \mathscr{H} u$ числа $r \in(0 ; R), \delta \in(0 ; r / 10]$ такие, что $r \leqslant\|a-x\| \leqslant r+\delta, x \in \partial X, a+\operatorname{int} \mathscr{B}_{r} \subset X$. Пусть $p$ - единичный вектор внешней нормали множества $X$ в точке $x$. Тогда

$$
\|a+r p-x\| \leqslant 3 \sqrt{r \delta} .
$$

ДОКАЗАТЕЛЬСТВО. Из условий леммы следует, что вектор $-p$ является единичным вектором внешней нормали множества $X^{c}$ в точке $x$. Применяя лемму 7 к множеству $X^{c}$, получим, что

$$
\left(x+R p+\operatorname{int} \mathscr{B}_{R}\right) \cap\left(x+\mathscr{B}_{R / 2}\right) \subset X^{c} .
$$

Отсюда и из включения $a+\operatorname{int} \mathscr{B}_{r} \subset X$ следует, что

$$
\left(x+R p+\operatorname{int} \mathscr{B}_{R}\right) \cap\left(x+\mathscr{B}_{R / 2}\right) \cap\left(a+\operatorname{int} \mathscr{B}_{r}\right)=\varnothing .
$$

Покажем, что

$$
\left(x+R p+\operatorname{int} \mathscr{B}_{R}\right) \cap\left(a+\operatorname{int} \mathscr{B}_{r}\right)=\varnothing .
$$

Предположим противное: существует вектор $z \in\left(x+R p+\operatorname{int} \mathscr{B}_{R}\right) \cap\left(a+\operatorname{int} \mathscr{B}_{r}\right)$. Применим лемму 8 для $u=x-a, v=z-a$. В силу этой леммы существует число $\theta \in(0 ; 1)$ такое, что для вектора $w=\theta u+(1-\theta) v$ справедливы неравенства $\|w\|<r$ и $\|u-w\|^{2}<2(r+\delta) \delta$.

Обозначим $y=\theta x+(1-\theta) z$. Так как $z \in x+R p+\operatorname{int} \mathscr{B}_{R}$, то $\|x+R p-z\|<R$. Поэтому

$\|x+R p-y\|=\|(1-\theta)(x+R p-z)+\theta R p\| \leqslant(1-\theta)\|x+R p-z\|+\theta R<(1-\theta) R+\theta R=R$.

Поскольку

$$
y-a=\theta(x-a)+(1-\theta)(z-a)=\theta u+(1-\theta) v=w,
$$

то $\|y-a\|=\|w\|<r$. Так как $y-x=(y-a)-(x-a)=w-u$, то

$$
\|y-x\|^{2}=\|u-w\|^{2}<2(r+\delta) \delta .
$$

Отсюда и из условий $r \in(0 ; R), \delta \in(0 ; r / 10]$ следует, что

$$
\|y-x\|^{2} \leqslant \frac{11}{50} r^{2}<\frac{r^{2}}{4}<\frac{R^{2}}{4}
$$

а значит, $\|y-x\|<R / 2$. Поэтому $y \in\left(x+R p+\operatorname{int} \mathscr{B}_{R}\right) \cap\left(x+\mathscr{B}_{R / 2}\right) \cap\left(a+\operatorname{int} \mathscr{B}_{r}\right)$, что противоречит условию (19). Тем самьм, доказано условие (20).

Из условия (20) следует, что $\|x+R p-a\| \geqslant R+r$. Применим лемму 1 для $x_{0}=x$, $x_{1}=x+R p, x_{2}=a, R_{1}=R, R_{2}=r$. Получим неравенство

$$
\|(R+r) x-R a-r(x+R p)\|^{2} \leqslant(R+r)(4 R r+(R+r) \delta) \delta .
$$

Следовательно,

$$
R^{2}\|a+r p-x\|^{2} \leqslant(R+r)(4 R r+(R+r) \delta) \delta \leqslant 2 R\left(4 R r+2 R \frac{r}{10}\right) \delta<9 R^{2} r \delta .
$$

Поэтому $\|a+r p-x\| \leqslant 3 \sqrt{r \delta}$. 
Лемма 10. Пусть в гильбертовом пространстве $\mathscr{H}$ множсество $X$ является телесно-гладким с константой $1 / R, R>0$. Пусть $p_{0}-$ единичный вектор внешней нормали множества $X$ в точке $x_{0} \in \partial X$. Тогда справедливо включение

$$
x_{0}-R p_{0}+\operatorname{int} \mathscr{B}_{R} \subset X .
$$

ДоКАЗАТЕЛЬСТвО. Для любого числа $r>0$ определим множество

$$
G(r)=x_{0}-r p_{0}+\mathscr{B}_{r}
$$

Пусть заданы числа $r, \widetilde{r}, 0<\widetilde{r}<r$, и вектор $x \in G(\widetilde{r})$. Тогда

$$
\left\|x_{0}-r p_{0}-x\right\| \leqslant\left\|x_{0}-\widetilde{r} p_{0}-x\right\|+\left\|r p_{0}-\widetilde{r} p_{0}\right\| \leqslant \widetilde{r}+r-\widetilde{r}=r .
$$

Следовательно, $x \in G(r)$. Тем самым, доказана монотонность многозначного отображения $G(r): G(\widetilde{r}) \subset G(r)$ при любых $r, \widetilde{r}$ таких, что $0<\widetilde{r}<r$.

Через $r_{0}$ обозначим супремум таких чисел $r>0$, что $G(r) \subset X$. Если $r_{0} \geqslant R$, то в силу определения супремума и монотонности $G(r)$ для любого $r \in(0 ; R)$ справедливо включение $G(r) \subset X$. Так как

$$
x_{0}-R p_{0}+\operatorname{int} \mathscr{B}_{R}=\bigcup_{r \in(0 ; R)} G(r)
$$

то в этом случае $x_{0}-R p_{0}+\operatorname{int} \mathscr{B}_{R} \subset X$, т.е. утверждение леммы справедливо.

Для завершения доказательства леммы достаточно показать, что $r_{0} \geqslant R$. Предположим противное: $r_{0}<R$.

В силу леммы 7 справедливо включение

$$
\left(x_{0}-R p_{0}+\operatorname{int} \mathscr{B}_{R}\right) \cap\left(x_{0}+\mathscr{B}_{R / 2}\right) \subset X .
$$

Отсюда и из включения $G(R / 4) \subset\left(x_{0}-R p_{0}+\operatorname{int} \mathscr{B}_{R}\right) \cap\left(x_{0}+\mathscr{B}_{R / 2}\right)$ следует, что $G(R / 4) \subset X$, а значит, $r_{0} \geqslant R / 4>0$.

В силу определения супремума получаем, что

$$
G\left(r_{0}+\frac{1}{k}\right) \not \subset X
$$

для любого $k \in \mathbb{N}$. Следовательно, существует последовательность векторов $\left\{z_{k}\right\}$ такая, что $z_{k} \in G\left(r_{0}+1 / k\right)$ и $z_{k} \notin X$ для любого $k \in \mathbb{N}$.

Так как $z_{k} \notin X$ и $x_{0}-r_{0} p_{0} \in X$, на отрезке с концами $x_{0}-r_{0} p_{0}$ и $z_{k}$ найдется точка $x_{k} \in \partial X$. Поскольку указанньй отрезок содержится в шаре $G\left(r_{0}+1 / k\right)$, то $x_{k} \in G\left(r_{0}+1 / k\right)$. Тем самым, доказано сушествование последовательности векторов $\left\{x_{k}\right\}$ таких, что $x_{k} \in G\left(r_{0}+1 / k\right) \cap \partial X$ для любого $k \in \mathbb{N}$.

Обозначим $a_{0}=x_{0}-r_{0} p_{0}$. Поскольку $x_{k} \in G\left(r_{0}+1 / k\right)$, то

$$
\left\|x_{0}-\left(r_{0}+\frac{1}{k}\right) p_{0}-x_{k}\right\| \leqslant r_{0}+\frac{1}{k} .
$$


Следовательно,

$$
\left\|a_{0}-x_{k}\right\| \leqslant\left\|x_{0}-\left(r_{0}+\frac{1}{k}\right) p_{0}-x_{k}\right\|+\frac{1}{k} \leqslant r_{0}+\frac{2}{k}
$$

Из неравенства $r_{0}>0$ следует существование номера $k_{0}$ такого, что $2 / k<r_{0} / 10$ при $k \geqslant k_{0}$. В силу определения супремума и монотонности $G(r)$ получаем, что $G(r) \subset X$ при $0<r<r_{0}$. Поэтому

$$
a_{0}+\operatorname{int} \mathscr{B}_{r_{0}}=\bigcup_{r \in\left(0 ; r_{0}\right)} G(r) \subset X
$$

Применяя лемму 9 для $a=a_{0}, r=r_{0}, x=x_{k}, \delta=2 / k, k \geqslant k_{0}$, получаем

$$
\left\|a_{0}+r_{0} p_{k}-x_{k}\right\| \leqslant 3 \sqrt{\frac{2 r_{0}}{k}} \rightarrow 0 \quad \text { при } k \rightarrow \infty
$$

где $p_{k}$ - единичные векторы внешних нормалей множества $X$ в точках $x_{k}$.

По условию леммы

$$
\left\|p_{k}-p_{0}\right\| \leqslant \frac{1}{R}\left\|x_{k}-x_{0}\right\|
$$

Следовательно,

$$
\begin{aligned}
\left\|x_{k}-x_{0}\right\| & \leqslant\left\|x_{k}-x_{0}+r_{0}\left(p_{0}-p_{k}\right)\right\|+r_{0}\left\|p_{k}-p_{0}\right\| \\
& \leqslant\left\|x_{k}-x_{0}+r_{0}\left(p_{0}-p_{k}\right)\right\|+\frac{r_{0}}{R}\left\|x_{k}-x_{0}\right\|
\end{aligned}
$$

Поэтому

$$
\left\|x_{k}-x_{0}\right\| \leqslant \frac{R}{R-r_{0}}\left\|x_{k}-x_{0}+r_{0}\left(p_{0}-p_{k}\right)\right\|=\frac{R}{R-r_{0}}\left\|x_{k}-a_{0}-r_{0} p_{k}\right\| .
$$

Отсюда и из соотношения (22) следует, что

$$
x_{k} \rightarrow x_{0} \quad \text { при } k \rightarrow \infty \text {. }
$$

Так как $r_{0}<R$, существует натуральное число $k_{1} \geqslant k_{0}$ такое, что $r_{0}+1 / k_{1}<R$. Тогда при $k \geqslant k_{1}$ справедливы включения $x_{k} \in G\left(r_{0}+1 / k\right) \subset x_{0}-R p_{0}+$ int $\mathscr{B}_{R}$. Из включения $x_{k} \in \partial X$ следует, что $x_{k} \notin$ int $X$. Из условия (21) следует включение

$$
\left(x_{0}-R p_{0}+\operatorname{int} \mathscr{B}_{R}\right) \cap\left(x_{0}+\operatorname{int} \mathscr{B}_{R / 2}\right) \subset \operatorname{int} X
$$

Поэтому $x_{k} \notin x_{0}+\operatorname{int} \mathscr{B}_{R / 2}$ при $k \geqslant k_{1}$. Следовательно, $\left\|x_{k}-x_{0}\right\| \geqslant R / 2$ при $k \geqslant k_{1}$, что противоречит соотношению (23).

ЛЕмма 11. Пусть в гильбертовом пространстве $\mathscr{H}$ множсество $X$ является телесно-гладким с константой $1 / R, R>0$. Тогда для любого вектора $x_{1} \in \operatorname{int} X$ существует вектор $d \in \mathscr{H}$ такой, что $x_{1} \in d+\operatorname{int} \mathscr{B}_{R} \subset \operatorname{int} X$. 
ДокАЗАТЕЛЬСТво. Определим число $r_{0}$ как супремум таких чисел $r>0$, что $x_{1}+$ $\mathscr{B}_{r} \subset X$. Тогда

$$
x_{1}+\operatorname{int} \mathscr{B}_{r_{0}}=\bigcup_{r \in\left(0 ; r_{0}\right)}\left(x+\mathscr{B}_{r}\right) \subset X,
$$

a значит, $x_{1}+\operatorname{int} \mathscr{B}_{r_{0}} \subset \operatorname{int} X$. Из условия $x_{1} \in \operatorname{int} X$ следует, что $r_{0}>0$.

Если $r_{0} \geqslant R$, то $x_{1}+\operatorname{int} \mathscr{B}_{R} \subset x_{1}+\operatorname{int} \mathscr{B}_{r_{0}} \subset \operatorname{int} X$. Тогда утверждение леммы выполнено для вектора $d=x_{1}$.

Рассмотрим случай $r_{0}<R$. Определим число $\delta=r_{0} / 10$. По определению супремума $x+\operatorname{int} \mathscr{B}_{r_{0}+\delta} \not \subset X$, т.е. существует вектор $z \in x_{1}+\operatorname{int} \mathscr{B}_{r_{0}+\delta}, z \notin X$. Так как $x_{1} \in X, z \notin X$, на отрезке с концами $x_{1}, z$ существует вектор $x_{0} \in \partial X$. При этом $r_{0}<\left\|x_{1}-x_{0}\right\| \leqslant\left\|x_{1}-z\right\|<r_{0}+\delta$.

Применим лемму 9 для $x=x_{0}, a=x_{1}, r=r_{0}$. Получим, что

$$
\left\|x_{1}+r_{0} p_{0}-x_{0}\right\| \leqslant 3 \sqrt{r_{0} \delta}=\frac{3 r_{0}}{\sqrt{10}}<r_{0},
$$

где $p_{0}-$ единичный вектор внешней нормали множества $X$ в точке $x_{0}$. Поэтому $x_{1} \in x_{0}-$ $r_{0} p_{0}+$ int $\mathscr{B}_{r_{0}} \subset x_{0}-R p_{0}+$ int $\mathscr{B}_{R}$.

В силу леммы 10 справедливо включение $x_{0}-R p_{0}+\operatorname{int} \mathscr{B}_{R} \subset \operatorname{int} X$. Полагая $d=x_{0}-R p_{0}$, получаем требуемое утверждение.

ЛЕмма 12. Пусть в гильбертовом пространстве $\mathscr{H}$ множсество $X$ является телесно-гладким с константой $1 / R, R>0$. Тогда $\operatorname{clint} X=\operatorname{cl} X$.

ДокАЗАТЕЛЬСтво. Предположим противное: существует точка $x_{0} \in \operatorname{cl} X, x_{0} \notin$ $\operatorname{clint} X$. Тогда $x_{0} \in \partial X$. В силу леммы 10 найдется вектор $p \in \mathscr{H},\|p\|=1, x_{0}-R p+$ int $\mathscr{B}_{R} \subset X$, а значит, $x_{0}-R p+\operatorname{int} \mathscr{B}_{R} \subset \operatorname{int} X$. Так как $x_{0} \in \operatorname{cl}\left(x_{0}-R p+\operatorname{int} \mathscr{B}_{R}\right)$, то $x_{0} \in \operatorname{clint} X$, что противоречит условию $x_{0} \notin \operatorname{clint} X$.

ТЕОРемА 3 (о связи слабой вьпуклости и гладкости множества). Пусть заданы число $R>0$ и множество $X$ в гильбертовом пространстве $\mathscr{H}$. Следующие два условия эквивалентны:

А) множество $X$ является телесно-гладким с константой $1 / R$;

В) мнохсество $\mathrm{cl} X$ слабо выпукло с константой $R$, множество int $X$ не пусто и слабо вогнуто с константой $R$, справедливы равенства

$$
\operatorname{int} \operatorname{cl} X=\operatorname{int} X, \quad \operatorname{clint} X=\operatorname{cl} X \text {. }
$$

ДокАЗАтЕльСтво. 1) Пусть вьполнено условие А). Тогда в силу леммы 11 для любого вектора $x \in \operatorname{int} X$ существует вектор $d \in \mathscr{H}$ такой, что $x \in d+\operatorname{int} \mathscr{B}_{R} \subset \operatorname{int} X$. Отсюда в силу предложения 3 следует слабая вогнутость множества int $X$ с константой $R$. Аналогично, применение леммы $11 \mathrm{k}$ множеству $X^{c}$ дает слабую вьпуклость множества $\mathrm{cl} X$ с константой $R$.

Применяя лемму 12 к множествам $X$ и $X^{c}$, получаем равенства $\operatorname{clint} X=\operatorname{cl} X$ и $\operatorname{clint}\left(X^{c}\right)=\operatorname{cl}\left(X^{c}\right)$, т.e. int $\operatorname{cl} X=\operatorname{int} X$. Тем самьм, доказано, что из условия А) следует условие В).

2) Пусть вьполнено условие В). Тогда в силу леммы 4 для любой точки $x \in \partial X$ существует вектор $p=p(x) \in \mathscr{H}$ такой, что

$$
\|p\|=1, \quad x+R p+\operatorname{int} \mathscr{B}_{R} \subset \operatorname{int}\left(X^{c}\right), \quad x-R p+\operatorname{int} \mathscr{B}_{R} \subset \operatorname{int} X .
$$


Покажем, что

$$
T_{X}(x)=\{v \in \mathscr{H}:\langle p, v\rangle \leqslant 0\} .
$$

Пусть $v \in \mathscr{H},\langle p, v\rangle \leqslant 0$. Тогда вектор $v$ принадлежит контингентному конусу шара $x-R p+\operatorname{int} \mathscr{B}_{R}$ в точке $x$. Отсюда и из включения $x-R p+\operatorname{int} \mathscr{B}_{R} \subset X$ следует, что $v \in T_{X}(x)$. Поэтому $\{v \in \mathscr{H}:\langle p, v\rangle \leqslant 0\} \subset T_{X}(x)$.

Пусть $v \in \mathscr{H}, v \notin\{v \in \mathscr{H}:\langle p, v\rangle \leqslant 0\}$, т.е. $\langle p, v\rangle>0$. Тогда вектор $v$ не принадлежит контингентному конусу множества $\left(x+R p+\operatorname{int} \mathscr{B}_{R}\right)^{c}$ в точке $x$. Из включения $x+R p+\operatorname{int} \mathscr{B}_{R} \subset \operatorname{int}\left(X^{c}\right)$ следует, что $X \subset\left(x+R p+\operatorname{int} \mathscr{B}_{R}\right)^{c}$. Поэтому $v \notin T_{X}(x)$. Следовательно, $T_{X}(x) \subset\{v \in \mathscr{H}:\langle p, v\rangle \leqslant 0\}$. Отсюда и из включения $\{v \in \mathscr{H}:\langle p, v\rangle \leqslant 0\} \subset T_{X}(x)$ следует равенство $(25)$.

Проводя аналогичные рассуждения для множества $X^{c}$, получим равенство

$$
T_{X^{c}}(x)=\{v \in \mathscr{H}:\langle p, v\rangle \geqslant 0\} .
$$

Поэтому для любого $x \in \partial X$ вектор $p=p(x)$ является единичным вектором внешней нормали множества $X$ в точке $x$.

Из условий (24) в силу леммы 5 следует, что функция $p: \partial X \rightarrow \mathscr{H}$ удовлетворяет условию Липшица с константой $1 / R$. Поэтому вьполнено условие A).

СлЕДСТВИЕ. Пусть заданы число $R>0$ и выпуклое множество $X$ в гильбертовом пространстве $\mathscr{H}$. Следующие условия әквивалентны:

А) множество $X$ является телесно-гладким с константой $1 / R$;

В) мнохество int $X$ не пусто и слабо вогнуто с константой $R$.

ДокАЗАТЕльство. Так как множество $\mathrm{cl} X$ вьпукло и замкнуто, то оно слабо выпукло с любой положительной константой. Из выпуклости множества $X$ и условия int $X \neq \varnothing$ следуют равенства int $\operatorname{cl} X=\operatorname{int} X, \operatorname{clint} X=\operatorname{cl} X$. Отсюда и из теоремы 3 получаем требуемое утверждение.

\section{4. Теорема о минимаксе.}

Лемма 13. Пусть в гильбертовом пространстве $\mathscr{H}$ заданы множсества $X, Y$. Пусть множество $X$ слабо выпукло с константой $R>0$, множество $Y$ сильно выпукло с константой $r \in(0 ; R)$. Тогда $X+Y \stackrel{*}{Y}=X$.

ДокАЗАТЕЛЬСтво. Так как множество $X$ слабо вьпукло с константой $R$, то

$$
X+\operatorname{int} \mathscr{B}_{R} * \operatorname{int} \mathscr{B}_{R}=X .
$$

\section{Поэтому}

$X \subset X+\mathscr{B}_{r} * \mathscr{B}_{r} \subset X+\mathscr{B}_{r}+\operatorname{int} \mathscr{B}_{R-r} *$ int $\mathscr{B}_{R-r} * \mathscr{B}_{r}=X+\operatorname{int} \mathscr{B}_{R} *$ int $\mathscr{B}_{R}=X$.

Следовательно, $X+\mathscr{B}_{r} * \mathscr{B}_{r}=X$. Поскольку множество $Y$ сильно выпукло с константой $r$, в силу предложения 8 существует множество $Y_{1} \subset \mathscr{H}$ такое, что $Y+Y_{1}=\mathscr{B}_{r}$. Поэтому

$$
X \subset X+Y \stackrel{*}{\subset} Y \subset+Y+Y_{1} * Y_{1} *{ }^{*}=X+\mathscr{B}_{r} * \mathscr{B}_{r}=X .
$$

Следовательно, $X+Y \stackrel{*}{Y}=X$. 
Лемма 14. Пусть в гильбертовом пространстве $\mathscr{H}$ заданы множества $X, Y$. Пусть множество $X$ слабо выпукло с константой $R_{1}>0$, множество int $X$ слабо вогнуто с константой $R_{2}>0, X=\operatorname{cl} i n t X$. Пусть множество $Y$ сильно выпукло с константой $R_{2}$. Тогда $X \stackrel{*}{*} Y+Y=X$.

ДокаЗАТельство. Покажем, что $X * \mathscr{B}_{R_{2}}+\mathscr{B}_{R_{2}}=X$. Если $x \in \operatorname{int} X$, то в силу слабой вогнутости множества int $X$ с константой $R_{2}$ согласно предложению 3 существует вектор $d \in \mathscr{H}$ такой, что $x \in d+\operatorname{int} \mathscr{B}_{R_{2}} \subset \operatorname{int} X$. Следовательно, $x \in d+\mathscr{B}_{R_{2}} \subset$ cl int $X=X$. Если $x \in \partial X$, то в силу леммы 4 существует вектор $p \in \mathscr{H}$ такой, что $\|p\|=1$ и $x-R_{2} p+\operatorname{int} \mathscr{B}_{R_{2}} \subset \operatorname{int} X$. Обозначим $d=x-R_{2} p$. Тогда $x \in d+\mathscr{B}_{R_{2}} \subset$ cl int $X=X$. Итак, мы доказали, что для любого вектора $x \in X$ существует вектор $d \in \mathscr{H}$ такой, что $x \in d+\mathscr{B}_{R_{2}} \subset X$. Из условия $d+\mathscr{B}_{R_{2}} \subset X$ следует, что $d \in X * \mathscr{B}_{R_{2}}$. Поэтому для любого $x \in X$ справедливо включение $x \in X * \mathscr{B}_{R_{2}}+\mathscr{B}_{R_{2}}$. Следовательно, $X \subset X \stackrel{*}{\mathscr{B}_{R_{2}}}+\mathscr{B}_{R_{2}}$. А значит, $X \stackrel{*}{*} \mathscr{B}_{R_{2}}+\mathscr{B}_{R_{2}}=X$.

Поскольку множество $Y$ сильно вьпукло с константой $R_{2}$, в силу предложения 8 сушествует множество $Y_{1} \subset \mathscr{H}$ такое, что $Y+Y_{1}=\mathscr{B}_{R_{2}}$. Поэтому

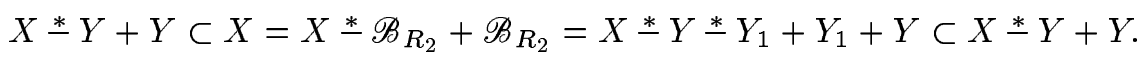

Следовательно, $X * * Y+Y=X$.

ТЕорема 4 (о перестановочности геометрических операций). Пусть в гильбертовом пространстве $\mathscr{H}$ заданы множества $X, Y, Z$. Пусть множество $X$ слабо выпукло с константой $R_{1}>0$, множество int $X$ слабо вогнуто с константой $R_{2}>0, X=\operatorname{clint} X$. Пусть множество $Y$ сильно выпукло с константой $r_{1} \in\left(0 ; R_{1}\right)$, множсество $Z$ сильно выпукло с константой $r_{2} \in\left(0 ; R_{2}\right)$. Тогда

$$
X+Y \stackrel{*}{Z}=X \stackrel{*}{*} Z+Y .
$$

ДокАЗАТЕЛЬСТво. Определим натуральное число $n$ из условия $r_{2} / n<R_{1}-r_{1}$. Для любого $k \in\{0,1, \ldots, n\}$ определим множество $Z_{k}=\frac{k}{n} Z$. Покажем, что для любого $k \in\{0,1, \ldots, n\}$ справедливо равенство

$$
X \stackrel{*}{*} Z_{k}+Y=X+Y \stackrel{*}{*} Z_{k} .
$$

При $k=0$ равенство (26) справедливо. Пусть равенство (26) справедливо при $k=s \in$ $\{0,1, \ldots, n-1\}$. Покажем, что равенство (26) справедливо при $k=s+1$.

В силу пункта A) предложения 7 множество $X * Z_{s+1}$ слабо вьпукло с константой $R_{1}$. Согласно пункту В) предложения 5 множество $Z_{s+1}$ сильно выпукло с константой $((s+1) / n) r_{2} \leqslant r_{2}$. Поэтому множество $Z_{s+1}$ сильно выпукло с константой $r_{2}$. Отсюда и из теоремы 1 следует, что множество $\operatorname{int}\left(X \stackrel{*}{*} Z_{s+1}\right)$ слабо вогнуто с константой $R_{2}-r_{2}$ и выполнено равенство $X * Z_{s+1}=\operatorname{cl} \operatorname{int}\left(X * Z_{s+1}\right)$. Отсюда в силу теоремы 2 получаем, что множество $W=X * Z_{s+1}+Y$ слабо вьпукло с константой $R_{1}-r_{1}$. Поскольку в силу пункта В) предложения 5 множество $Z_{1}=\frac{1}{n} Z$ сильно выпукло с константой $r_{2} / n<R_{1}-r_{1}$, в силу леммы 13 справедливо равенство $W+Z_{1} * Z_{1}=W$, т.е.

$$
X \stackrel{*}{*} Z_{s+1}+Y+Z_{1} * Z_{1}=X * Z_{s+1}+Y .
$$

Согласно пункту А) предложения 7 множество $X * Z_{s}$ слабо вьпукло с константой $R_{1}$. Из теоремы 1 следует, что множество $\operatorname{int}\left(X * Z_{s}\right)$ слабо вогнуто с константой $R_{2}-$ 
$(s / n) r_{2} \geqslant(1 / n) r_{2}$ и выполнено равенство $X * Z_{s}=\operatorname{cl} \operatorname{int}\left(X * Z_{s}\right)$. Отсюда и из сильной выпуклости множества $Z_{1}$ с константой $(1 / n) r_{2}$ согласно лемме 14 получаем равенство $X \stackrel{*}{*} Z_{s} * Z_{1}+Z_{1}=X \stackrel{*}{ } Z_{s}$. Следовательно,

$$
X \stackrel{*}{ } Z_{s}+Y^{*} Z_{1}=X \stackrel{*}{*} Z_{s} * Z_{1}+Z_{1}+Y^{*} Z_{1}=X \stackrel{*}{ } Z_{s+1}+Y+Z_{1} \stackrel{*}{ } Z_{1} \text {. }
$$

Отсюда и из равенства (27) получаем равенство

$$
X \stackrel{*}{ } Z_{s}+Y \stackrel{*}{*} Z_{1}=X^{*} Z_{s+1}+Y .
$$

Поскольку равенство (26) выполняется при $k=s$, то $X * Z_{s}+Y=X+Y \stackrel{*}{*} Z_{s}$. Следовательно,

$$
X+Y \stackrel{*}{*} Z_{s+1}=X+Y^{*} Z_{s} \stackrel{*}{ } Z_{1}=X \stackrel{*}{ } Z_{s}+Y \stackrel{*}{*} Z_{1}=X \stackrel{*}{ } Z_{s+1}+Y,
$$

т.е. равенство (26) вьполняется при $k=s+1$. По индукции получаем справедливость равенства (26) для любого $k \in\{0,1, \ldots, n\}$. Применяя равенство (26) для $k=n$, получаем требуемое утверждение.

Заметим, что требование $r_{1}<R_{1}$ в условиях теоремы 4 нельзя заменить на неравенство $r_{1} \leqslant R_{1}$. Пусть, например, $\mathscr{H}=\mathbb{R}, X=(-\infty ; 0] \cup[1 ;+\infty), Y=Z=[0 ; 1]$. Тогда множество $Y=Z$ сильно выпукло с константой $r_{1}=r_{2}=1$, множество $X$ слабо выпукло с константой $R_{1}=1$, множество int $X$ слабо вогнуто с любой положительной константой $R_{2}$. Так как $X+Y=\mathbb{R}, X+Y$ * $Z=\mathbb{R}, X$ * $Z=(-\infty ;-1] \cup[1 ;+\infty)$, $X \stackrel{*}{Z}+Y=X$, равенство $X+Y *{ }^{*} Z=X * Z+Y$ не выполняется.

Лемма 15. Пусть в линейном пространстве $\mathscr{L}$ заданы множества $X, P, Q$ и функиия $f: \mathscr{L} \rightarrow \mathbb{R}$. Пусть для любого вектора $x_{0} \in X$ достигается минимум по $u \in P$ функиии

$$
u \mapsto \sup _{v \in Q} f\left(x_{0}-u+v\right) .
$$

Тогда следующие условия эквивалентны:

А) для любого $x_{0} \in X$ справедливо равенство

$$
\sup _{v \in Q} \inf _{u \in P} f\left(x_{0}-u+v\right)=\inf _{u \in P} \sup _{v \in Q} f\left(x_{0}-u+v\right)
$$

В) для любого $\lambda \in \mathbb{R}$ справедливо равенство

$$
\left(M_{\lambda}+P \stackrel{*}{ } Q\right) \cap X=\left(M_{\lambda} * \text { } Q+P\right) \cap X,
$$

где $M_{\lambda}$ - лебегово множество функиии $f: M_{\lambda}=\{x \in \mathscr{L}: f(x) \leqslant \lambda\}$. 
ДокАЗАТЕльство. 1) Предположим, что условие В) не выполнено. Тогда, поскольку включение $M_{\lambda} * Q+P \subset M_{\lambda}+P$ * $Q$ справедливо всегда, существует вектор $x_{0} \in X$ такой, что $x_{0} \in M_{\lambda}+P * Q$ и $x_{0} \notin M_{\lambda} * Q+P$. Поэтому для любого вектора $v \in Q$ существует вектор $u \in P$ такой, что $x_{0}-u+v \in M_{\lambda}$, и для любого вектора $u \in P$ существует вектор $v \in Q$ такой, что $x_{0}-u+v \notin M_{\lambda}$. Следовательно, для любого вектора $v \in Q$ справедливо неравенство

$$
\inf _{u \in P} f\left(x_{0}-u+v\right) \leqslant \lambda
$$

и для любого вектора $u \in P$ справедливо неравенство

$$
\sup _{v \in Q} f\left(x_{0}-u+v\right)>\lambda \text {. }
$$

Отсюда следует, что

$$
\sup _{v \in Q} \inf _{u \in P} f\left(x_{0}-u+v\right) \leqslant \lambda
$$

и, поскольку достигается

$$
\min _{u \in P}\left(\sup _{v \in Q} f\left(x_{0}-u+v\right)\right),
$$

то

$$
\inf _{u \in P} \sup _{v \in Q} f\left(x_{0}-u+v\right)>\lambda \text {. }
$$

Поэтому условие А) не выполнено.

2) Теперь предположим, что условие А) не вьполнено. Тогда, поскольку неравенство

$$
\sup _{v \in Q} \inf _{u \in P} f\left(x_{0}-u+v\right) \leqslant \inf _{u \in P} \sup _{v \in Q} f\left(x_{0}-u+v\right)
$$

выполнено всегда, существует вектор $x_{0} \in X$ и число $\lambda$ такие, что

$$
\sup _{v \in Q} \inf _{u \in P} f\left(x_{0}-u+v\right)<\lambda<\inf _{u \in P} \sup _{v \in Q} f\left(x_{0}-u+v\right) .
$$

Поэтому для любого вектора $v \in Q$ существует вектор $u \in P$ такой, что $f\left(x_{0}-u+v\right)<\lambda$, и для любого вектора $v \in Q$ существует вектор $u \in P$ такой, что $f\left(x_{0}-u+v\right)>\lambda$. Следовательно, $x_{0} \in M_{\lambda}+P *{ }^{*} Q$ и $x_{0} \notin M_{\lambda} * Q+P$. Это означает, что условие В) не выполнено.

Из теорем 3,4 и леммы 15 следует

ТЕорема 5 (о минимаксе). Пусть в гильбертовом пространстве $\mathscr{H}$ заданы множества $X, P, Q$ и функиия $f: \mathscr{H} \rightarrow \mathbb{R}$. Пусть для любого вектора $x_{0} \in X$ достигается минимум по $и \in P$ функиии

$$
u \mapsto \sup _{v \in Q} f\left(x_{0}-u+v\right) .
$$

Пусть для любого числа $\lambda \in \mathbb{R}$ лебегово множество $M_{\lambda}=\{x \in \mathscr{H}: f(x) \leqslant \lambda\}$ замкнуто и является телесно-гладким с константой $1 / R, R>0$. Пусть множсества $P, Q$ сильно выпуклы с константой $r \in(0 ; R)$. Тогда для любого вектора $x_{0} \in \mathscr{H}$ справедливо равенство

$$
\sup _{v \in Q} \inf _{u \in P} f\left(x_{0}-u+v\right)=\inf _{u \in P} \sup _{v \in Q} f\left(x_{0}-u+v\right) .
$$

Если в выражении (28) инфимум и супремум достигаются, то равенство (28) означает существование седловой точки в рассматриваемой минимаксной задаче. 
5. Теорема об альтернативе для дифференциальных игр. Рассмотрим дифференциальную игру

$$
\frac{d x}{d t}=v(t)-u(t), \quad x(0)=x_{0}
$$

на отрезке времени $t \in[0 ; T]$ с терминальным множеством $M \subset \mathbb{R}^{n}$, где $x(t), u(t), v(t) \in$ $\mathbb{R}^{n}, x(t)$ - фазовьй вектор системы, $u(t)$ - управление игрока-преследователя, $v(t)-$ управление игрока-убегающего. Управления игроков подчиняются геометрическим ограничениям:

$$
u(t) \in P(t), \quad v(t) \in Q(t) \quad \text { при почти всех } t \in[0 ; T],
$$

где $P, Q$ - заданные многозначные отображения, значениями которых являются множества $P(t) \subset \mathbb{R}^{n}, Q(t) \subset \mathbb{R}^{n}$.

Цель игрока-преследователя состоит в приведении фазового вектора в конечньй момент времени на терминальное множество: $x(T) \in M$. Цель игрока-убегающего - противоположная: $x(T) \notin M$.

Если при некоторой реализации игры выполнилось условие $x(T) \in M$, то будем говорить, что произошла поимка. В противном случае будем говорить, что произошло уклонение.

ОПРЕДЕЛЕНИЕ. Через $\mathscr{U}$ будем обозначать множество допустимых программных стратегий игрока-преследователя, состоящее из всех интегрируемых по Лебегу функций $u:[0 ; T] \rightarrow \mathbb{R}^{n}$, удовлетворяющих условию $u(t) \in P(t)$ при почти всех $t \in[0 ; T]$. Через $\mathscr{V}$ будем обозначать множество допустимых программных стратегий игрока-убегающего, состоящее из всех интегрируемых по Лебегу функций $v:[0 ; T] \rightarrow \mathbb{R}^{n}$, удовлетворяющих условию $v(t) \in Q(t)$ при почти всех $t \in[0 ; T]$.

ОПрЕДЕЛЕниЕ. Пусть заданы $u \in \mathscr{U}, v \in \mathscr{V}, x_{0} \in \mathbb{R}^{n}$. Через $x_{T}(u, v)$ обозначим значение фазового вектора в конечный момент времени $T$ при заданных управлениях игроков $u, v$ и заданном начальном значении фазового вектора $x_{0}$ :

$$
x_{T}(u, v)=x_{0}-\int_{0}^{T} u(t) d t+\int_{0}^{T} v(t) d t .
$$

ОПРЕДЕЛЕНИЕ. Пусть на отрезке $[a ; b]$ задано многозначное отображение $X$, значениями которого являются множества $X(t) \subset \mathbb{R}^{n}$. Интегралом Аумана $\int_{a}^{b} X(t) d t$ назьвается множество векторов $\int_{a}^{b} x(t) d t$, где $x$ - интегрируемые по Лебегу функции, удовлетворяющие условию $x(t) \in X(t)$ почти всюду на $[a ; b]$.

ТЕорема 6 (об альтернативе). Пусть в дифференииальной игре (29), (30) терминальное множество $M$ замкнуто и является телесно-гладким с константой $1 / R, R>0$. Пусть интегралы Аумана

$$
P_{0}=\int_{0}^{T} P(t) d t \quad u \quad Q_{0}=\int_{0}^{T} Q(t) d t
$$

являются сильно выпуклыми множсествами с константой $r \in(0 ; R)$. Тогда для любого вектора $x_{0} \in \mathbb{R}^{n}$ выполнено одно и только одно из условий: либо

А) существует программная стратегия игрока-преследователя $u \in \mathscr{U}$ такая, что для любой программной стратегии игрока-убегающего $v \in \mathscr{V}$ происходит поимка: $x_{T}(u, v) \in M$, либо 
В) существует программная стратегия игрока-убегающего $v \in \mathscr{V}$ такая, что для любой программной стратегии игрока-преследователя $u \in \mathscr{U}$ происходит уклонение: $x_{T}(u, v) \notin M$.

А именно, если $x_{0} \in M * Q_{0}+P_{0}$, то выполняется условие $\left.\mathrm{A}\right)$, иначе выполняется условие В).

ДокАЗАТЕЛьСтво. Рассмотрим случай $x_{0} \in M * Q_{0}+P_{0}$. В этом случае существует вектор $u_{0} \in P_{0}$ такой, что $x_{0} \in M * Q_{0}+u_{0}$. Поэтому $x_{0}-u_{0}+Q_{0} \subset M$. Следовательно, для любого вектора $v_{0} \in Q_{0}$ справедливо включение $x_{0}-u_{0}+v_{0} \in M$. Отсюда и из определения интеграла Аумана следует, что существует стратегия $u \in \mathscr{U}$ такая, что для любой стратегии $v \in \mathscr{V}$ происходит поимка:

$$
x_{T}(u, v)=x_{0}-\int_{0}^{T} u(t) d t+\int_{0}^{T} v(t) d t \in M .
$$

Рассмотрим обратный случай: $x_{0} \notin M * Q_{0}+P_{0}$. В силу теоремы 3 множество $M$ является слабо выпуклым с константой $R$, множество int $M$ является слабо вогнутьм c константой $R$ и $\operatorname{clint} M=\mathrm{cl} M$. Поэтому согласно теореме 4 справедливо равенство $M * Q_{0}+P_{0}=M+P_{0} * Q_{0}$. Следовательно, в рассматриваемом случае вьполнено условие $x_{0} \notin M+P_{0} * Q_{0}$. Поэтому $x_{0}+Q_{0} \not \subset M+P_{0}$. Это означает, что сушествует вектор $v_{0} \in Q_{0}$ такой, что для любого вектора $u_{0} \in P_{0}$ справедливо условие $x_{0}+v_{0}-u_{0} \notin M$. Иными словами, существует стратегия $v \in \mathscr{V}$ такая, что для любой стратегии $u \in \mathscr{U}$ происходит уклонение: $x_{T}(u, v) \notin M$.

\section{СПИСОК ЦИТИРОВАННОЙ ЛИТЕРАТУРЫ}

[1] Половинкин Е. С., Балашов М. В. Элементы выпуклого и сильно выпуклого анализа. М.: Физматлит, 2004.

[2] Иванов Г. Е., Половинкин Е. С. О сильно выпуклых линейных дифференциальных играх // Дифференц. уравнения. 1995. Т. 31. №10. С. 1641-1648.

[3] Иванов Г. Е. Непрерывность оптимальных управлений в дифференциальных играх и некоторые свойства слабо и сильно выпуклых функций // Матем. заметки. 1999. Т. 66. №6. C. $816-839$.

[4] Иванов Г.Е. Гладкость и выпуклость маргинальных функций // Некоторые проблемы фундаментальной и прикладной математики. Сб. научных трудов. М.: Московский физикотехнический институт, 1999. С. 79-92.

Московский физико-технический институт

Поступило

E-mail: givanov@math.mipt.ru

16.01.2004 